\title{
Cell Intrinsic Galectin-3 Attenuates Neutrophil ROS-Dependent Killing of Candida by Modulating CR3 Downstream Syk Activation
}

\author{
Sheng-Yang Wu', Juin-Hua Huang ${ }^{1}$, Wen-Yu Chen ${ }^{1}$, Yi-Chen Chan², Chun-Hung Lin'², \\ Yee-Chun Chen ${ }^{3}$, Fu-Tong Liü and Betty A. Wu-Hsieh ${ }^{4 *}$ \\ 'Graduate Institute of Immunology, National Taiwan University College of Medicine, Taipei, Taiwan, ${ }^{2}$ Institute of Biological \\ Chemistry, Academia Sinica, Taipei, Taiwan, ${ }^{3}$ Department of Internal Medicine, National Taiwan University Hospital, Taipei, \\ Taiwan, ${ }^{4}$ Institute of Biomedical Sciences, Academia Sinica, Taipei, Taiwan
}

OPEN ACCESS

Edited by:

Silvia Mercedes Uriarte,

University of Louisville, USA

Reviewed by:

Hridayesh Prakash,

University of Hyderabad, India

Marisa Mariel Fernandez,

University of Buenos Aires, Argentina

*Correspondence:

Betty A. Wu-Hsieh

bwh@ntu.edu.tw

Specialty section:

This article was submitted to

Microbial Immunology,

a section of the journal

Frontiers in Immunology

Received: 02 November 2016

Accepted: 12 January 2017

Published: 03 February 2017

Citation:

Wu S-Y, Huang J-H, Chen W-Y,

Chan $Y-C$, Lin $C-H$, Chen $Y-C$,

Liu F-T and Wu-Hsieh BA (2017) Cell

Intrinsic Galectin-3 Attenuates

Neutrophil ROS-Dependent Killing

of Candida by Modulating CR3

Downstream Syk Activation.

Front. Immunol. 8:48.

doi: 10.3389/fimmu.2017.00048
Invasive candidiasis is a leading cause of nosocomial bloodstream infection. Neutrophils are the important effector cells in host resistance to candidiasis. To investigate the modulation of neutrophil fungicidal function will advance our knowledge on the control of candidiasis. While recombinant galectin-3 enhances neutrophil phagocytosis of Candida, we found that intracellular galectin-3 downregulates neutrophil fungicidal functions. Co-immunoprecipitation and immunofluorescence staining reveal that cytosolic gal3 physically interacts with Syk in neutrophils after Candida stimulation. Ga/3 ${ }^{-1-}$ neutrophils have higher level of Syk activation as well as greater abilities to generate reactive oxygen species (ROS) and kill Candida than ga/3//+ cells. While galectin-3 deficiency modulates neutrophil and macrophage activation and the recruitment of monocytes and dendritic cells, the deficiency does not affect the numbers of infiltrating neutrophils or macrophages. Galectin-3 deficiency ameliorates systemic candidiasis by reducing fungal burden, renal pathology, and mortality. Adoptive transfer experiments demonstrate that cell intrinsic galectin-3 negatively regulates neutrophil effector functions against candidiasis. Reducing galectin-3 expression or activity by siRNA or gal3 inhibitor TD139 enhances human neutrophil ROS production. Mice treated with TD139 have enhanced ability to clear the fungus. Our work unravels the mechanism by which galectin-3 regulates Sykdependent neutrophil fungicidal functions and raises the possibility that blocking gal3 in neutrophils may be a promising therapeutic strategy for treating systemic candidiasis.

Keywords: galectin-3, Candida albicans, neutrophil, reactive oxygen species, Syk kinase, TD139, M2 macrophage, monocyte/dendritic cell recruitment

\section{INTRODUCTION}

Galectin-3 (gal3) belongs to a family of evolutionarily highly conserved animal lectins. It is structurally unique amongst all the known galectins by consisting of a proline- and glycine-rich non-lectin domain in the $\mathrm{N}$-terminal part, in addition to the $\mathrm{C}$-terminal carbohydrate-recognition domains (CRD) (1). Gal3 binds to the $N$-acetyllactosamine motif of glycoproteins through its CRD and undergoes polymerization through its $\mathrm{N}$-terminal part $(1,2)$. It is expressed in macrophages, 
dendritic cells, activated lymphocytes, epithelial cells, and neutrophils and found in both intracellular and extracellular compartments as well as on the cell surface (3). Recombinant gal3 has been reported to function extracellularly as a chemoattractant, a pro-apoptosis mediator, an opsonizing molecule, a phagocytosis enhancer, and a mediator that bridges hyperglycosylated mucin MUC2 and dendritic cells (2). Cell intrinsic gal3 has also been reported to function intracellularly as an anti-apoptosis mediator and a regulator for $\mathrm{T}$ cell activation, keratinocyte migration, and phagocyte cellular responses (4-8). Intracellular gal3 modulates cellular responses and tumor cell apoptosis by direct association with internalized dectin-1 (9), signaling molecule (10), adaptor protein (11), or apoptosis regulator (12). Point mutation at the CRD abolishes gal3 binding to Bax, thereby interfering its antiapoptotic effect (12), showing that the CRD of gal3 is involved in interaction with proteins, but the interaction is carbohydrate independent.

Neutrophils express gal3 intracellularly although at lower levels compared to other innate cells $(3,13)$. In vitro studies showed that gal3 added exogenously improves the ability of neutrophils to migrate, phagocytose, and produce IL- 8 and reactive oxygen species (ROS) (13-15). Studies of the role of intracellular gal3 in neutrophil functions are limited. By comparing $\mathrm{gal3}^{-/-}$to $\mathrm{gal3}^{+/+}$ cells, gal3 is found to inhibit the neutrophil ROS response to phorbol myristate acetate or zymosan stimulation and regulate the life span of neutrophils upon Toxoplasma gondii infection (4). Thus, it appears that exogenously added gal3 and intrinsic gal3 have distinct effects on neutrophils.

Candida albicans is a commensal organism colonizing human mucosal surface and the skin. The commensal organism grows as yeast and germinates to invade the host (16). Neutropenic patients or individuals with neutrophil dysfunction are at higher risk for invasive candidiasis (17-19). Invasive candidiasis affects more than 250,000 people each year and leads to more than 50,000 deaths worldwide. Mortality among patients with invasive candidiasis can be as high as $40 \%$ even after receiving antifungal therapy (20). Study on candidemia cases revealed that time to initiate antifungal therapy has no impact on 30-day mortality (21), demonstrating that most patients do not respond to antifungal treatment. To develop better diagnostic biomarkers for patients with candidemia and better therapeutic strategy for disseminated candidiasis are important issues. In a mouse model of systemic candidiasis, Candida has marked tropism for kidney and brain (22). Mice with X-linked chronic granulomatous disease or with myeloperoxidase deficiency whose neutrophils are defective in producing ROS succumb to sublethal Candida infection (23). Depleting neutrophils exacerbates systemic candidiasis (24). These reports support the notion that neutrophils through producing ROS are responsible for clearing Candida infection in both humans and mice. The animal model of systemic candidiasis is a powerful tool to investigate the function of neutrophils against Candida infection. Whether and how cell intrinsic gal3 regulates host defense against candidemia has never been addressed.

While most studies on the role of gal3 in Candida emphasize its extracellular functions, we uncover its intracellular role in modulating signaling pathways. Extracellular gal3 mostly sends outside-in signals to regulate cell activation, we show a direct regulation of Syk kinase by cytosolic gal3 in neutrophil response to Candida. Gal3 physically interacts with Syk and negatively regulates its activation. By applying cell-permeable gal3 inhibitor TD139 on human neutrophils or administering it to mice with systemic candidiasis, our work raises the possibility that blockade of gal3 in neutrophils may be a promising therapeutic strategy for systemic Candida infection.

\section{MATERIALS AND METHODS}

\section{Fungus and Infection}

Candida albicans strain SC5314 (ATCC MYA-2876) and clinical isolates from patients with bloodstream infection (CL 10-1, CA 09-1, CL 15-1, CA 01-1, CL 03-1, and CA 03-1, National Taiwan University Hospital) were used in this study. SC5314, clinical isolates, and GFP-expressing strain OG1 (25) were cultured at $30^{\circ} \mathrm{C}$ on yeast-peptone-dextrose (YPD) agar (DIFCO) plate. Mice were injected intravenously with Candida yeasts prepared in RPMI 1640 medium. Candida yeasts were opsonized in phenol red free HBSS containing $10 \%$ fresh mouse or human serum at room temperature for $30 \mathrm{~min}$.

\section{Mouse Neutrophil Enrichment and Adoptive Transfer}

Bone marrow cells were harvested from the femurs and suspended in APBS buffer before overlaid on discontinuous percoll gradients $(55,62$, and $81 \%$ in the order from top to bottom) (GE Healthcare). After centrifugation at $1,400 \times g$ for $30 \mathrm{~min}$, cells at the interface between 62 and $81 \%$ gradients were harvested and washed. Flow cytometric analysis showed that $92 \%$ of cells were $\mathrm{CD} 11 \mathrm{~b}^{+} \mathrm{Ly}_{6 \mathrm{G}}{ }^{+}$. For adoptive transfer, $5 \times 10^{6}$ neutrophils isolated from $\mathrm{gal3}^{+/+}, \mathrm{gal3}^{-/-}$, and littermate mice were injected into recipient mice through the tail vein. Recipient mice were given Candida intravenously $1 \mathrm{~h}$ later.

\section{Human Neutrophil Isolation}

Fresh peripheral venous bloods were collected from healthy volunteers with no history of candidiasis in BD Vacutainer ${ }^{\circledR}$ Plus Plastic K2EDTA Tubes or serum separator tubes for different purposes. Neutrophils were isolated as described previously (26). Briefly, bloods were diluted in $\mathrm{Mg}^{2+}$ - and $\mathrm{Ca}^{2+}$-free DPBS and overlaid on Ficoll-Paque solution (Ficoll-Paque ${ }^{\mathrm{TM}}$ PLUS, GE Healthcare). After centrifugation at $600 \times g$ for $15 \mathrm{~min}$, the pellet containing neutrophils and red blood cells (RBCs) was subject to dextran sedimentation (3\%) to separate RBCs from neutrophils. Residual RBCs were lysed by lysis buffer. Cell viability was determined by trypan blue exclusion.

\section{Staining Surface and Cytosolic Gal3}

Neutrophils from human or mice were stimulated with opsonized Candida for 5 and $15 \mathrm{~min}$. Cells were stained with rat anti-gal 3 and fluorochrome-conjugated goat anti-rat antibodies. After fixation in 4\% PFA and permeabilization in saponin (Sigma)-containing perm/wash buffer, cells were stained with Alexa 647-rat anti-gal3 antibody. Samples were acquired by BD FACSCanto II and analyzed by BD FACSDiva software. 


\section{Renal Infiltrating Cell Isolation and Immunostaining}

Kidneys were cut into small pieces and treated with $0.2 \mathrm{mg} /$ $\mathrm{ml}$ Liberase $\mathrm{TM}$ (Roche) at $37^{\circ} \mathrm{C}$ for $30 \mathrm{~min}$. Pellets were resuspended in $45 \%$ percoll and overlaid on $81 \%$ percoll. Cells at the interface $(45 / 81 \%)$ were collected after centrifugation. Cells stained positive for CD45 were taken as infiltrating cells. After exclusion of $\mathrm{Ly} 6 \mathrm{G}^{+}$neutrophils, cells were divided into three distinct populations according to the expression of MHC II and F4/80 (MHC $\mathrm{II}^{+} \mathrm{F} 4 / 80^{+}$as macrophages, $\mathrm{MHC} \mathrm{II}^{-} \mathrm{F} 4 / 80^{+}$as monocytes, and $\mathrm{MHC} \mathrm{II}^{+} \mathrm{F} 4 / 80^{-}$as dendritic cells). Detailed description is given in Methods in Supplementary Material.

\section{ROS Production}

Bone marrow neutrophils were incubated in phenol red free HBSS containing $10 \mu \mathrm{M}$ CM- $\mathrm{H}_{2}$ DCFDA (Life Technologies) for 30 min. After replenishment with fresh HBSS or with HBSS containing kinase inhibitors, cells were stimulated with opsonized Candida yeasts $(\mathrm{MOI}=0.5)$. Oxidative DCF was analyzed by flow cytometry. To assess ROS production by renal infiltrating neutrophils isolated from infected mice, neutrophils collected from percoll gradients were stained with $\mathrm{CM}-\mathrm{H}_{2} \mathrm{DCFDA}$ and subject to flow cytometric analysis.

Human neutrophils were pretreated with mock (0.5\% DMSO) or $250 \mu \mathrm{M}$ of TD139 (3,3'-deoxy-3,3-bis(4-[m-fluorophenyl]1H-1,2,3-triazol-1-yl)-thio-digalactoside) (27) for $4 \mathrm{~h}$ before addition of CM- $\mathrm{H}_{2}$ DCFDA. Cells were then stimulated by opsonized Candida for 20 min before subject to flow cytometric analysis.

\section{Fungicidal Activity Assay}

The assay was adopted from Methods in Molecular Biology (28). Briefly, Candida yeasts were grown in Sabouraud-dextrose broth $(\mathrm{pH}=5.6)$ in $37^{\circ} \mathrm{C}$ overnight. Bone marrow neutrophils and $\mathrm{CD}^{2} 5^{+} \mathrm{Ly} \mathrm{G}^{+}$renal infiltrating cells were subject to killing assay in 96-well plate with $5 \times 10^{5}$ cells and $1 \times 10^{4}$ opsonized Candida yeasts. Wells containing opsonized Candida yeasts without neutrophils was used as control. Twenty minutes was allowed for phagocytosis after which supernatants in control wells and experimental groups were plated on YPD plate. The number of ingested yeasts $\left(N_{0 h}\right)=$ the number of yeasts in control well - the number of uningested yeasts. The rest of the wells were incubated for another $1.5 \mathrm{~h}$ and the cells were lysed to release intracellular yeasts. The lysates were plated. \% Killing $=\left(N_{0 \mathrm{~h}}-N_{1.5 \mathrm{~h}}\right) /$ $N_{\mathrm{oh}} \times 100 \%$, where $N=$ the number of intracellular Candida.

\section{Co-Immunoprecipitation}

Five million neutrophils were stimulated with opsonized Candida. Cells were lysed in $500 \mu \mathrm{l}$ of non-denaturing lysis buffer. Cell lysates were mixed with $1.5 \mu \mathrm{g}$ of mouse anti-gal3 antibody (B2C10) or $1 \mu \mathrm{g}$ of anti-Syk polyclonal antibody at $4^{\circ} \mathrm{C}$ overnight. The mixture was mixed with $50 \mu$ of agarose beads slurry under rotary agitation at $4^{\circ} \mathrm{C}$ for $4 \mathrm{~h}$. Lysate beads mixture was washed with IP washing buffer. Beads were then mixed with $25 \mu \mathrm{l}$ of sample buffer and boiled to elute immunoprecipitates.

\section{Quantification of Fungal Load}

Kidney and brain were homogenized in a tissue grinder with $1 \mathrm{ml}$ of RPMI 1640 medium. One to ten serial dilutions were made and $0.1 \mathrm{ml}$ was plated on YPD agar. Colonies were counted after incubation at $30^{\circ} \mathrm{C}$ for 2 days.

\section{Lgals3 Gene Knockdown in Human Neutrophils}

Three million human neutrophils were premixed with Nucleofector ${ }^{\circledast}$ Solution (Lonza) plus supplement in total volume of $100 \mu \mathrm{l}$ followed by addition of $2 \mu \mathrm{M}$ of scrambled siRNA or siRNA targeting gal3 (Santa Cruz Biotechnology). Cells were transfected by using Nucleofector ${ }^{\circledast}$ Program Y-001 in the nucleofector II device and incubated in RPMI complete medium for $7 \mathrm{~h}$ before further experiment. Knockdown efficiency was about $47 \%$. Cell viability was determined by trypan blue exclusion.

\section{Statistics}

Mann-Whitney test (non-parametric analysis) was used to compare the difference between two groups. Mortality after Candida infection was analyzed by log-rank and Fisher's exact test. Statistical significance was defined as $p<0.05$.

\section{Ethics Statement}

Human study was approved by the Institutional Review Board of the National Taiwan University Hospital (Permit Number: 201103121IRB, 201401074INA). All written informed consents were received from participants prior to inclusion in this study. Mouse study was carried out in strict accordance with the recommendations in the Guidebook for the Care and Use of Laboratory Animals, The Third Edition, 2007, published by The Chinese-Taipei Society of Laboratory Animal Sciences. The experimental protocol was approved by the Committee on the Ethics of Animal Experiments of the National Taiwan University College of Medicine (Permit Number: 20120031, 20130238, and 20140304).

\section{RESULTS}

\section{Dynamics of gal3 Expression after Neutrophils Encountering Opsonized Candida}

Previous studies revealed that recombinant gal3 positively regulates neutrophil functions when added exogenously, here we focused our study on the role of cell intrinsic gal3 in neutrophils. Fluorescence microscopic (Figure 1A) and flow cytometric analyses (Figures 1B,C) showed that above $80 \%$ of unstimulated neutrophils expressed low levels of gal3 in the cytosol and stimulation with opsonized Candida increased intracellular gal3 expression as early as 5 min after stimulation (Figure 1B). While dendritic cells and monocytes/macrophages constitutively express gal 3 on the surface $(29,30)$, gal3 was not detected on the surface of unstimulated neutrophils (Figure 1C). Stimulation by Candida induced gal3 redistribution to the cell surface in about $20 \%$ of neutrophils (Figure 1C). It was noted that intracellular gal3 was diffusely distributed (Figure 1D) and $70.0 \pm 8.8 \%$ 
A

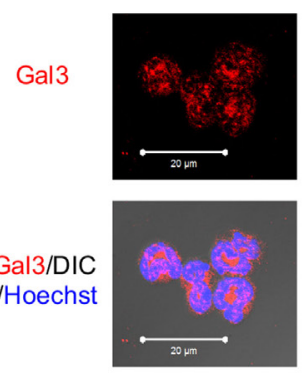

D
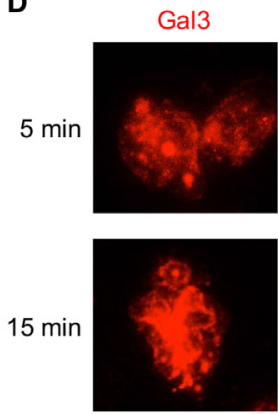

$5 \mathrm{~min}$
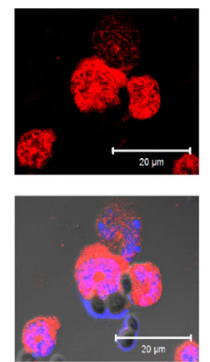

Ca/Hoechst
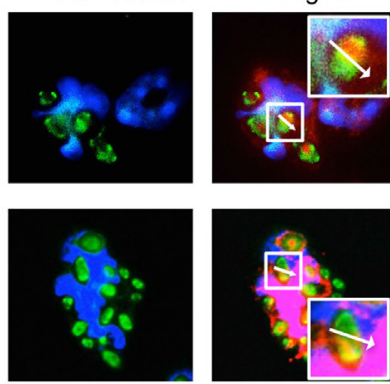

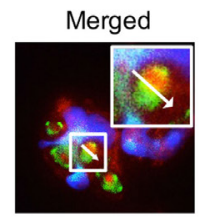

B

Cytosolic gal3
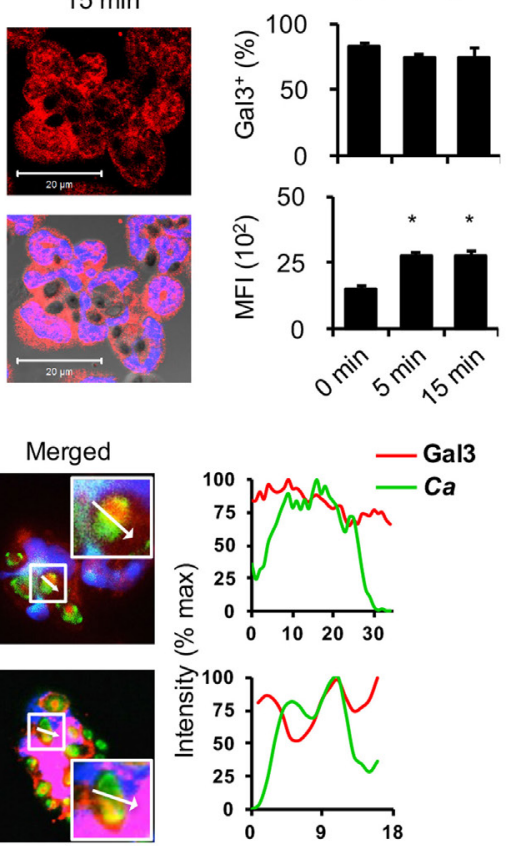

Distance (pixel)
C Surface gal3
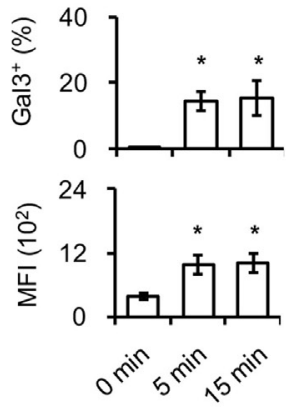

E

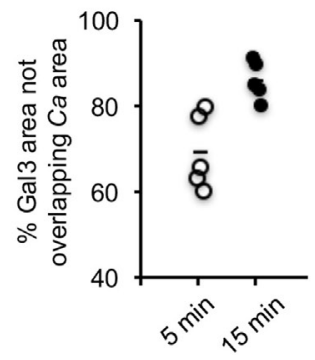

FIGURE 1 | Opsonized Candida upregulates cytosolic gal3 expression in neutrophils. Bone marrow neutrophils were stimulated with opsonized Candida SC5314 (A-C) or GFP+ OG1 (D,E) for 5 or 15 min. Zero minutes represent unstimulated cell controls incubated in serum-containing medium for 15 min. (A) Cells were cytospun, permeabilized, and stained for gal3 (red) and nucleus (blue) before confocal microscopic analysis for gal3 expression. DIC, differential interference contrast. (B,C) Neutrophils were stained with rat anti-gal3 and PE-goat anti-rat antibodies, fixed in 4\% PFA, permeabilized, and then stained with Alexa 647-rat anti-gal3 antibody. The \% of cells expressing cytosolic gal3+ $\mathbf{( B )}$ and surface gal3+ $\mathbf{( C )}$ in total cell population are shown in upper pane/s. The levels of cytosolic (B) and surface gal3 (C) expression are shown as mean fluorescence intensity (MFI) in lower panels. Data are presented as mean \pm SD. ${ }^{*} p<0.05$, as analyzed by Mann-Whitney test. $n=3$. (D,E) Neutrophils were allowed to ingest GFP+ OG1 Candida (green) and stained for gal3 (red) and nucleus (blue) before subjecting to fluorescence microscopic analysis. (D) The intensity of fluorochromes along the white arrow in the merged image is shown in the histogram on the right. (E) The \% of gal $3^{+}$area (red) that is not overlapped with Candida (green) in the whole gal $3^{+}$area (red) $\left(180,760 \mu \mathrm{m}^{2}\right.$ in average) at 5 and 15 min after encounter was analyzed by Metamorph software. Each dot represents one photo image. $n=5$.
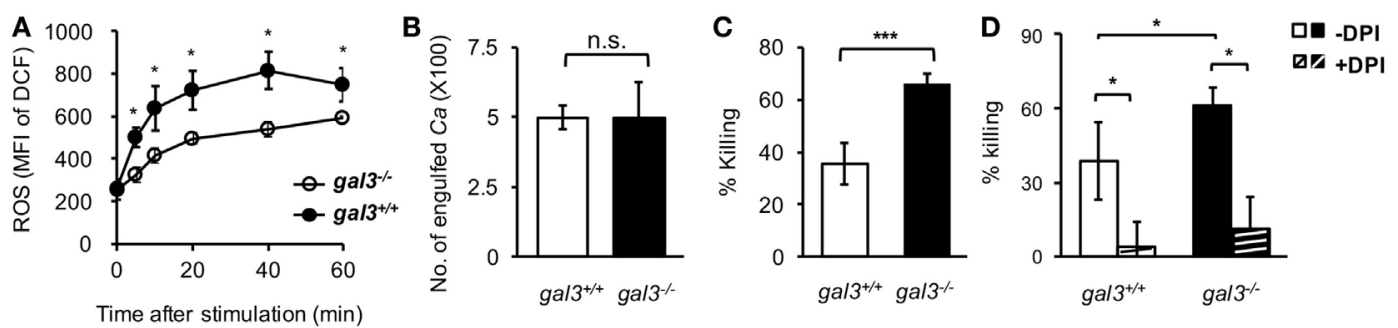

FIGURE 2 | Gal3 negatively regulates complement receptor 3 downstream reactive oxygen species (ROS)-dependent killing of Candida by

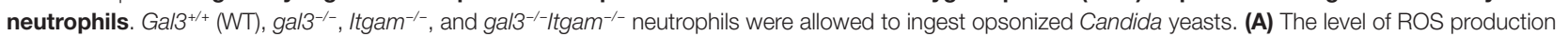
shown as mean fluorescence intensity (MFI) of oxidized DCF fluorescence. Zero minutes represent the MFI of unstimulated control incubated in serum-containing medium. $n=7$ pooled from two independent experiments in panel (A). (B) Twenty minutes was allowed for phagocytosis. The number of $\mathrm{Candida}(\mathrm{Ca})$ engulfed by neutrophils is shown as CFU. (C,D) The ability of neutrophils to kill opsonized Candida is shown as \% killing. Neutrophils were treated with or without NADPH inhibitor diphenyleneiodonium (DPI, $10 \mu \mathrm{M}$ ) for 30 min before addition of opsonized yeasts (D). DPI was left in the culture over the entire period. $n=7-8$ pooled from three independent experiments in panels (B,C); and $n=3$ in panel (D). Data are presented as mean \pm SD. ${ }^{\star} p<0.05$; ${ }^{\star \star *} p<0.005$; n.s., not significant, as analyzed by Mann-Whitney test. See also Figure S1 in Supplementary Material.

and $85.9 \pm 4.6 \%$ of the intracellular gal 3 at 5 and 15 min time points, respectively, did not colocalize with opsonized Candida (Figures 1D,E). By tracking the movement of cytosolic gal3 in neutrophils upon encountering GFP-expressing Candida, we found that gal3 moved toward engulfed Candida (Figure S1 in Supplementary Material). At the point when gal3 was closest to Candida (7.56 min), it still did not associate with the organism (Figure S1 and Video 1 in Supplementary Material). These 
results together indicate that stimulation by opsonized Candida upregulates gal3 expression in neutrophils and that intracellular gal3 does not bind to the engulfed organism.

\section{Gal3 Negatively Regulates Complement Receptor 3 (CR3) Downstream ROS-Dependent Killing of Candida in Neutrophils}

To unravel the role of upregulated gal3 in neutrophils, $\mathrm{gal}^{+/+}$and $\mathrm{gal3}^{-/-}$neutrophils were allowed to ingest opsonized Candida, and the subsequent cellular response was assessed. We found that $\mathrm{gal3}^{-/-}$neutrophils produced higher levels of ROS than $\mathrm{gal3}^{+/+}$ cells (Figure 2A). While gal3 ${ }^{-/-}$and gal3 $^{+/+}$cells were comparable in their abilities to engulf Candida (Figure 2B), gal3 $^{-/-}$neutrophils killed ingested Candida more efficiently than $\mathrm{gal}^{+/+}$cells (Figure 2C). Moreover, suppressing ROS completely abolished killing of ingested Candida yeasts by both $\mathrm{gal3}^{+/+}$and $\mathrm{gal3}^{-/-}$ neutrophils (Figure 2D). These results indicate that both $\mathrm{gal3}^{+/+}$ and gal3 $^{-/-}$neutrophils kill ingested Candida through a ROSdependent mechanism and that gal3 suppresses neutrophil fungicidal activity through inhibiting ROS production. Interestingly, CR3-deficient $\left(\right.$ Itgam $\left.^{-/}\right)$neutrophils were significantly less efficient in producing ROS (Figure 3A) and killing of opsonized Candida (Figure 3B) than WT cells, but such functions were not affected in dectin-1-deficient $\left(\mathrm{clec} 7 \mathrm{a}^{-/-}\right)$neutrophils (Figures
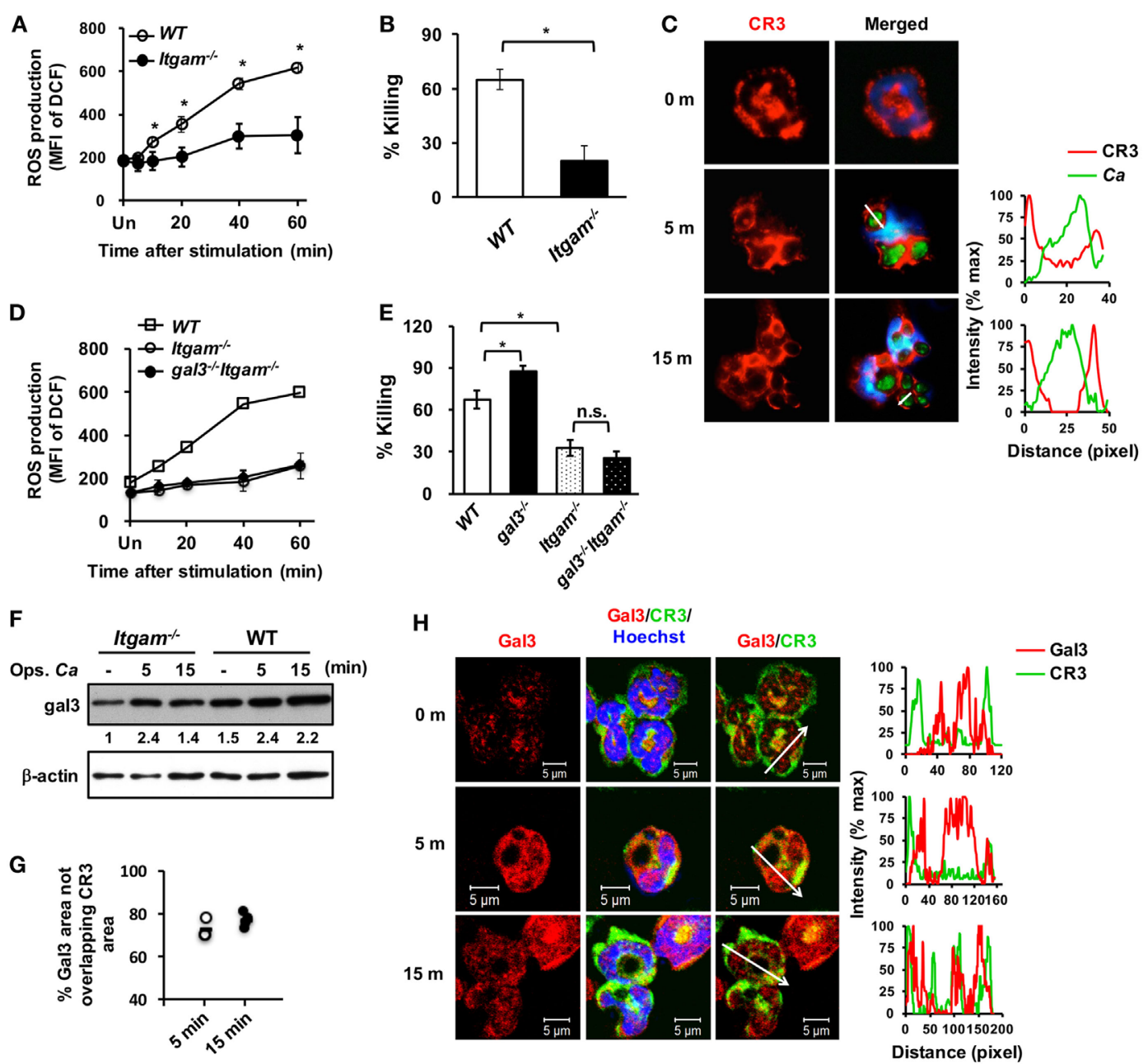

FIGURE 3 | Gal3 negatively regulates complement receptor 3 (CR3)-mediated killing of Candida by neutrophils. Neutrophils isolated from WT, ga/3-/, Itgam ${ }^{-/-}$, gal/-/-Itgam ${ }^{-1-}$ mice were allowed to ingest opsonized Candida yeasts. (A,D) The level of reactive oxygen species (ROS) production is shown as mean fluorescence intensity (MFI) of oxidized DCF fluorescence. $n=3$ in panel (A); $n=4$ in panel (D). Experiment was performed twice. (B,E) The ability of neutrophils to kill opsonized Candida is shown as \% killing. $n=3$ in panel (B); and $n=4$ in panel (E). Experiment was performed twice. Data are presented as mean \pm SD. ${ }^{*} p<0.05$; n.s., not significant, as analyzed by Mann-Whitney test. (C) Confocal fluorescence microscopic analysis to localize CR3 (red) and Candida OG1 (green). (F) Gal3 expression in Itgam ${ }^{-1-}$ and WT neutrophils stimulated with opsonized Candida (Ops. Ca) was detected by Western blot. $\beta$-actin was used as a loading control. The numbers below the gel denote the relative intensity of gal3. Experiment was performed three times. (G,H) Confocal microscopy analysis to localize gal3 (red) and CR3 (green). The intensity of different fluorochromes along the white arrow in the merged (C) and Gal3/CR3 (H) images is shown in the histogram on the right. (G) Metamorph software was employed to determine the percentages of non-localization between gal3 and CR3 in the whole gal3 area in image in panel (H) $\left(56,444 \mu \mathrm{m}^{2}\right.$ in average). Each dot represents one photo. $n=4$. 
S2A,B in Supplementary Material). Immunofluorescence staining revealed that CR3 congregated around but did not colocalize with engulfed Candida (Figure 3C). Thus, it appears that ROS production as a result of CR3 engagement is responsible for neutrophil fungicidal activity. In addition, gal3 deficiency promoted the abilities of dectin-1-deficient neutrophils to produce ROS (Figure S2C in Supplementary Material) and kill Candida (Figure S2D in Supplementary Material), whereas the presence or absence of gal3 did not make any difference in ROS production (Figure 3D) and the ability to kill Candida by Itgam $^{-/-}$cells (Figure 3E). It reveals that the suppressive effect of gal3 on neutrophil anti-Candida function is through CR3. It is worth noting that gal3 expression increased by twofold in Itgam $^{-/}$neutrophils after stimulation by opsonized Candida compared to that without stimulation (Figure 3F), revealing that gal3 upregulation is independent of CR3. Moreover, we found that majority $(>72 \%)$ of the intracellular gal3 in the cell population we tracked did not colocalize with CR3 (Figures 3G,H). These results together suggest that although neutrophil production of ROS is through CR3 engagement with Candida, gal3 does not directly interact with CR3.

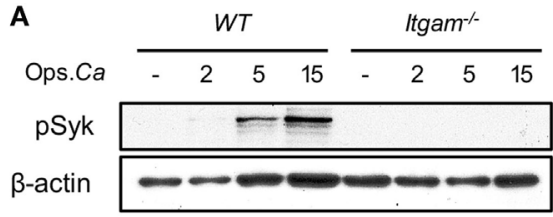

C

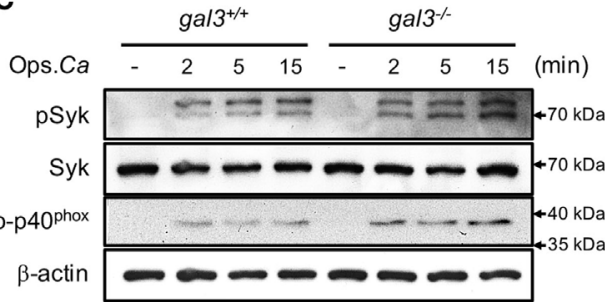

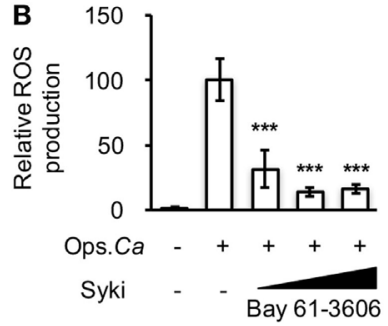

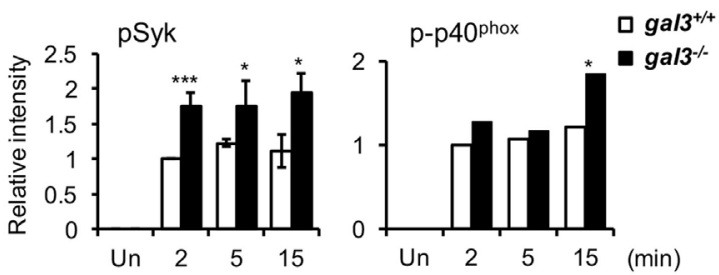

D Co-IP

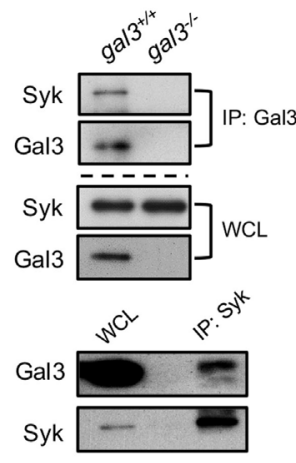

E

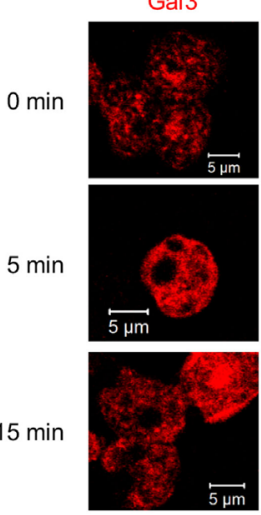

Syk
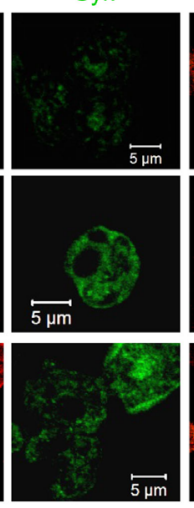

Merged
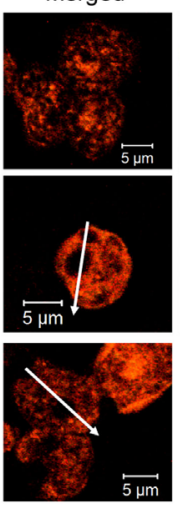

Merged/ DIC/Hoechst
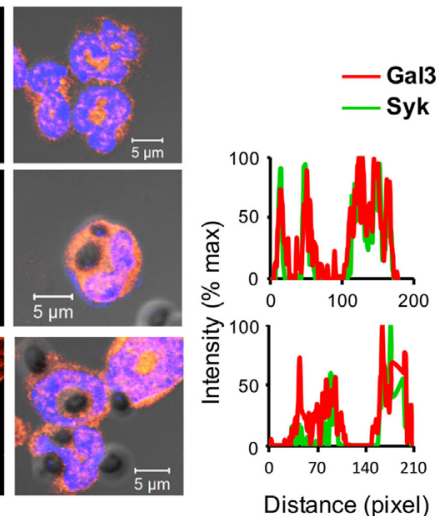

FIGURE 4 | Gal3 negatively regulates complement receptor 3 downstream Syk-mediated neutrophil reactive oxygen species (ROS) response to

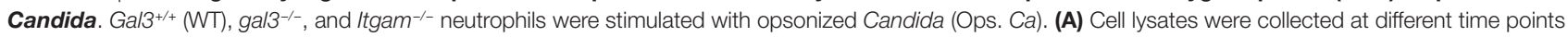
after stimulation and subjected to Western blot analysis with anti-p-Syk antibody. $\beta$-actin was used as a loading control. (B) WT cells were pretreated with 2, 10, and $50 \mu \mathrm{M}$ of Syk inhibitor before stimulation. The levels of ROS produced by neutrophils at 20 min after stimulation were analyzed by flow cytometry. Relative ROS production was calculated by dividing mean fluorescence intensity of oxidized DCF fluorescence in Candida-stimulated cells by that in untreated cells. $n=6$ as pooled from two independent experiments. (C) Cell lysates were collected at different time points after stimulation and subjected to Western blot analysis with anti-p-Syk, -Syk, or -p-p40 phox antibodies. $\beta$-actin was used as a loading control. Relative intensity of $p$-Syk $(n=5)$ and p-p40phox $(n=2)$ at different time points are shown in the right panel. Data are presented as mean \pm SEM. ${ }^{\star} p<0.05$, ${ }^{\star \star \star} p<0.005$, as analyzed by Mann-Whitney test. (D) Whole cell lysates (WCL) were immunoprecipitated with anti-gal3 (IP: Gal3, upper panel) and anti-Syk (IP: Syk, lower pane/) antibodies. Precipitates were subjected to Western blot analysis with anti-Syk and anti-gal3 antibodies. Experiments were performed three times. Data from one representative experiment are shown. (E) Confocal microscopy image of gal3 (red) and Syk (green) in neutrophils after stimulation by Candida. The intensity of different fluorochromes along the white arrow in the merged image is shown in the histogram on the right. See also Figure S1 in Supplementary Material. 


\section{Gal3 Physically Interacts with Syk and Downregulates CR3 Downstream Syk-Mediated ROS Production}

It is reported that CR3 engagement activates neutrophil killing of Candida through Syk signaling (31). Our data showed that the levels of pSyk in CR3-deficient neutrophils were completely abolished after stimulation (Figure 4A) while that in dectin1-deficient cells were comparable to WT cells (Figure S2E in Supplementary Material). Treatment with Syk inhibitor Bay 61-3606 dose-dependently reduced neutrophil ROS production (Figure 4B). Importantly, the levels of pSyk and p-p40 phox were significantly higher in $\mathrm{gal3}^{-/-}$than in $\mathrm{gal3}^{+/+}$neutrophils after stimulation (Figure 4C). Reciprocal co-immunoprecipitation (Figure 4D) and immunofluorescence staining (Figure 4E) revealed that gal3 directly interacted with Syk after stimulation.
Therefore, ROS production by neutrophils in response to opsonized Candida is Syk-dependent and gal3, by modulating Syk activation, regulates CR3 downstream signaling pathway(s) leading to ROS production.

\section{Gal3 Deficiency Enhances the Fungicidal Activity of Renal Infiltrating Neutrophils}

Previous studies revealed the role of gal 3 in cell recruitment in vivo $(32,33)$. To address whether gal3 also regulates cell infiltration in systemic candidiasis, we analyzed renal infiltrating neutrophils $\left(\mathrm{CD}^{4} 5^{+} \mathrm{Ly}_{6 \mathrm{G}}{ }^{+}\right)$, macrophages $\left(\mathrm{CD} 45^{+} \mathrm{Ly}_{6 \mathrm{G}}{ }^{-} \mathrm{M}\right.$ $\left.\mathrm{HCII}^{+} \mathrm{F} 4 / 80^{+}\right)$, monocytes $\left(\mathrm{CD}_{4} 5^{+} \mathrm{Ly}_{6 \mathrm{G}^{-}} \mathrm{MHCII}^{-} \mathrm{F} 4 / 80^{+}\right)$, and dendritic cell $\left(\mathrm{CD} 45^{+} \mathrm{Ly}_{6 \mathrm{G}^{-}} \mathrm{MHCII}^{+} \mathrm{F} 4 / 80^{-}\right)$populations (34). The results showed that gal3 deficiency did not affect the recruitment of neither neutrophils nor macrophages (Figures 5A-C).

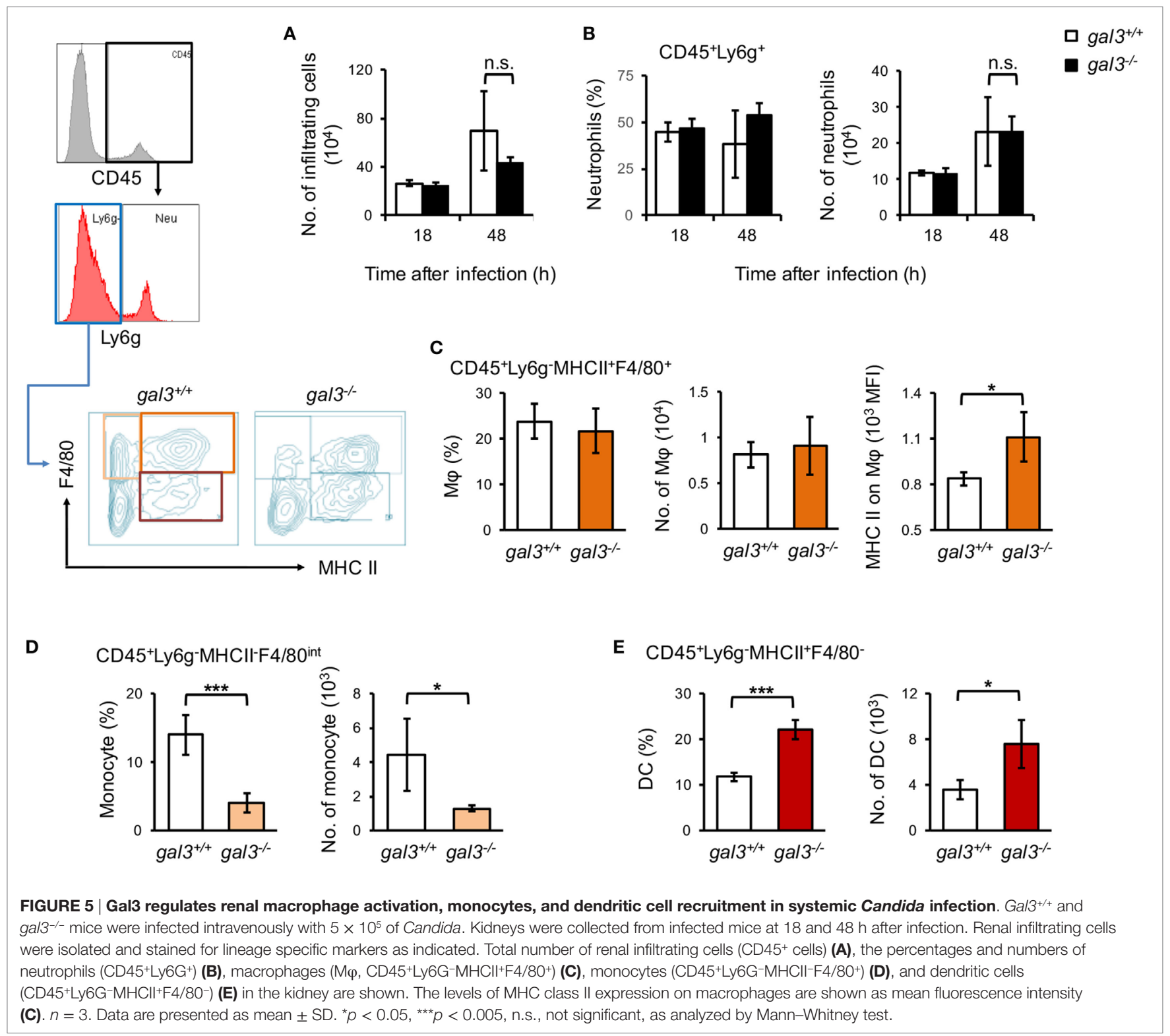


Gal3 deficiency, however, reduced monocyte (Figure 5D; Figure S4A in Supplementary Material) but enhanced dendritic cell (Figure 5E; Figure S4A in Supplementary Material) infiltration. In addition, the levels of chemokines were not different between $\mathrm{gal3}^{-/-}$and $\mathrm{gal3}^{+/+}$mice after infection (Figure S3 in Supplementary Material). Interestingly, we found that renal infiltrating macrophages in gal3 $^{-/}$mice expressed higher levels of MHC II (Figure 5C), iNOS, and lower CD206 than in $\mathrm{gal3}^{+/+}$ mice (Figure S4B in Supplementary Material).

Peripheral blood neutrophils from infected $\mathrm{gal3}^{-/-}$were compared to those from gal3 $^{+/+}$mice for the levels of activation marker CD11b (35). Results in Figure 6A showed that CD11b on neutrophils from $\mathrm{gal3}^{-/-}$mice were significantly higher than cells from $\mathrm{gal}^{+/+}$mice after infection (Figure 6A). Moreover, renal infiltrating neutrophils of infected gal3 $^{-/}$mice produced significantly higher amount of ROS (Figure 6B) and their ability to kill Candida (Figure 6C) was higher than cells of $\mathrm{gal3}^{+/+}$ mice. These results demonstrated that gal 3 negatively regulates neutrophil functions not only in vitro but also in vivo.

\section{Gal3/-- Mice Are More Resistant to Systemic Candida Infection}

Consistent with higher neutrophil fungicidal functions, $\mathrm{gal3}^{-/-}$ mice had better survival (Figure 7A) and lower fungal burdens in kidneys and brain (Figure 7B) than gal3 $^{+/+}$mice when systemically infected with Candida. While $\mathrm{gal3}^{+/+}$mice sustained multiple abscesses in the kidney with their kidney weights being about 2.3-fold of that of uninfected mice, gal $^{-/-}$mice exhibited only minimal gross renal pathology and relatively less change in kidney weights (Figure 7C). The creatinine and blood urea nitrogen levels were also significantly lower in $\mathrm{gal3}^{-/-}$mice than in gal3 $^{+/+}$mice (Figure 7D). Both gal $^{+/+}$and gal3 $^{-/-}$mice exhibited chronic-active nephritis and pyelonephritis with inflammatory infiltrates on day 2 after infection. Starting on day 3, nephritis became severe in $\mathrm{gal}^{+/+}$mice, but remained minimal/slight in gal3 $^{-/-}$mice (Figure 7E), indicating that severe inflammation and tissue damage occurred to $\mathrm{gal}^{+/+}$but not to $\mathrm{gal}^{-/-}$mice. Additionally, while hyphal/pseudohyphal forms of Candida were found in both infected $\mathrm{gal}^{+/+}$and $\mathrm{gal3}^{-/-}$mice on $18 \mathrm{~h}$ after infection, these forms increased and persisted in $\mathrm{gal}^{+/+}$mice, but became almost completely absent in $\mathrm{gal3}^{-/-}$mice by 3 days after infection (Figure S5 in Supplementary Material). These results together show that gal3 deficiency ameliorates systemic candidiasis by reducing fungal burdens, renal pathology, and mortality.

\section{Neutrophil Intrinsic gal3 Suppresses Host Defense against Candida}

Adoptive transfer experiments were performed to investigate whether gal3 regulates neutrophil functions in vivo is a cell intrinsic event. We found that adoptively transferred $\mathrm{gal}^{-/-}$neutrophils cleared Candida infection more efficiently than gal $^{+/+}$ neutrophils whether the recipient mice were $\mathrm{gal}^{+/+}$(Figure 8A) or gal3 $^{-/-}$(Figure 8B). Transfer of neutrophils from littermate donors also demonstrated the suppressive effect of gal 3 on neutrophils in clearing Candida (Figure 8C). When neutrophil cytosolic factor 1-deficient $\left(n c f-1^{-/}\right)$mice were used as recipients, transfer of $\mathrm{gal3}^{-/-}$neutrophils also resulted in significantly less fungal burdens than transfer of gal3 $^{+/+}$cells (Figure 8D). The results confirm that it is ROS produced by transferred neutrophils but not that by cells in the recipient mice that mediates host defense against Candida. Taken together, these results indicate that cell intrinsic gal3 regulates neutrophil anti-Candida function.

\section{Gal3 Negatively Regulates Primary Human Neutrophil ROS Response to Opsonized Candida}

Human neutrophils were like mouse neutrophils (Figure 1A) constitutively expressed gal 3 in the cytosol but not on the surface (Figure 9A). Surface gal3 became detectable as early as $5 \mathrm{~min}$ after encountering opsonized Candida (Figure 9A). Interestingly, silencing Lgals3 in primary human neutrophils by siRNA significantly enhanced ROS production (Figure 9B). Treatment with gal3 inhibitor TD139, a derivative of thiodigalactoside, augmented human neutrophil ROS production (Figure 9C) and reduced fungal burden in mice with systemic candidiasis (Figure 9D). These results demonstrate that gal3 is a negative regulator of human neutrophil ROS response and point to the possibility of employing TD139 as a treatment strategy to restore neutrophil functions against Candida infection.
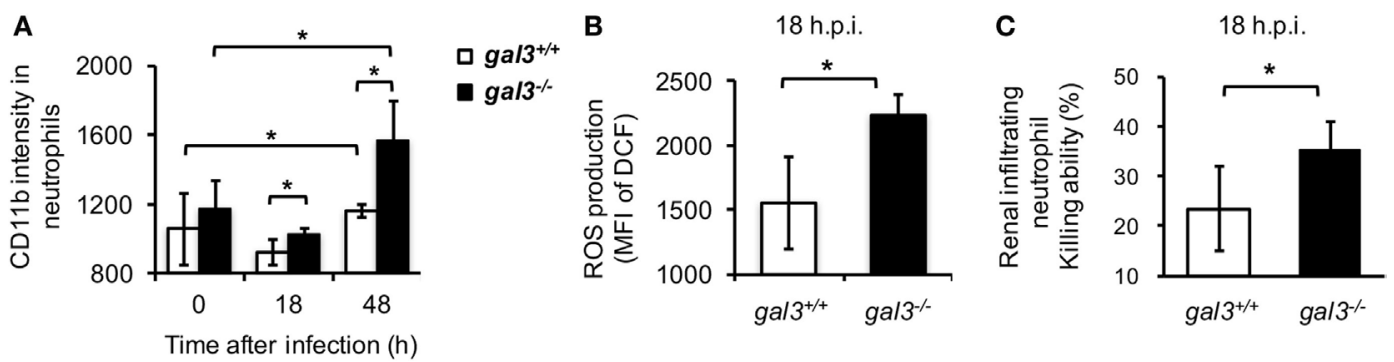

FIGURE 6 | Gal3 deficiency enhances infiltrating neutrophil anti-Candida functions. Ga/3//+ and ga/3//- mice were infected intravenously with $5 \times 10^{5}$ of Candida. (A) Blood was collected from naïve mice (0) or mice at $18 \mathrm{~h}$ and $48 \mathrm{~h}$ after infection. The levels of CD11b expression on CD45+CD11 $+\mathrm{Ly} 6 \mathrm{G}^{+}$peripheral blood neutrophils are presented as mean fluorescence intensity (MFI). $n=3$. (B,C) CD45+CD11 b+Ly6G+ cells were sorted from total infiltrating cells in the kidney at $18 \mathrm{~h}$ after infection. The levels of reactive oxygen species (ROS) production $\mathbf{( B )}$ and their ability to kill $\mathbf{( C )}$ were determined. $n=3$. Data are presented as mean \pm SD. ${ }^{*} p<0.05$, as analyzed by Mann-Whitney test. 

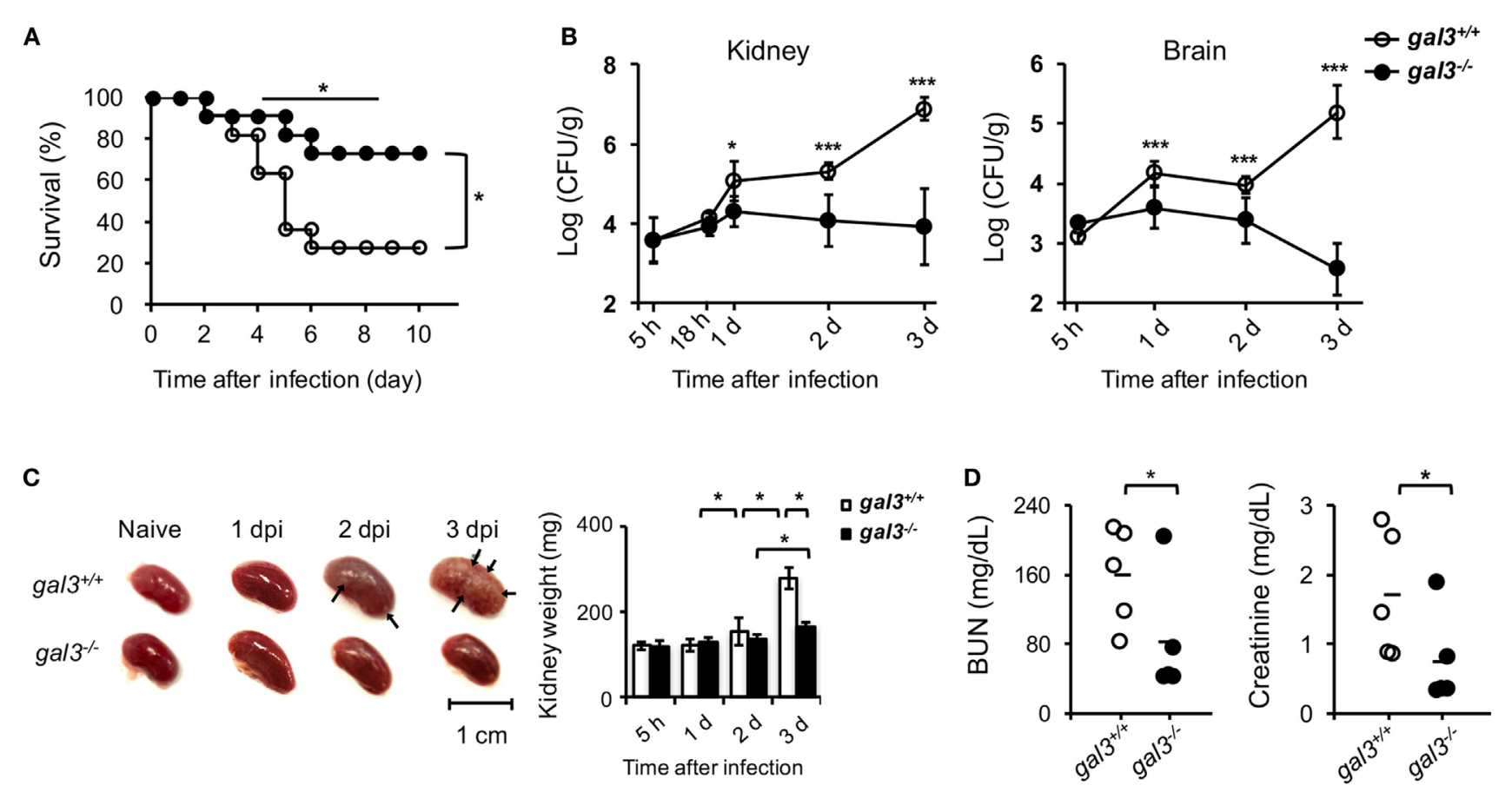

E

Naive

$2 \mathrm{dpi}$
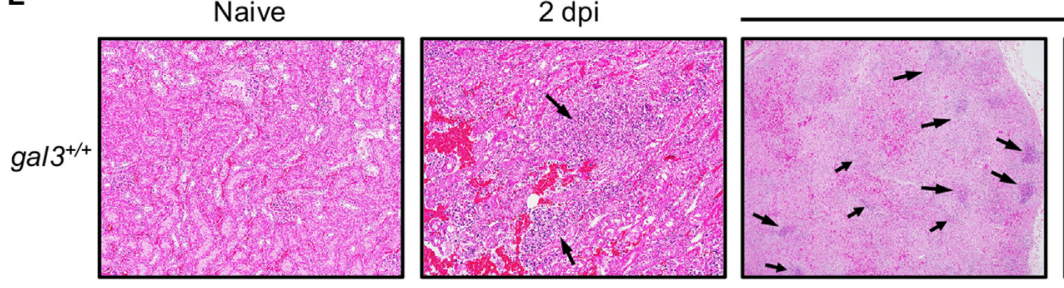

$3 \mathrm{dpi}$
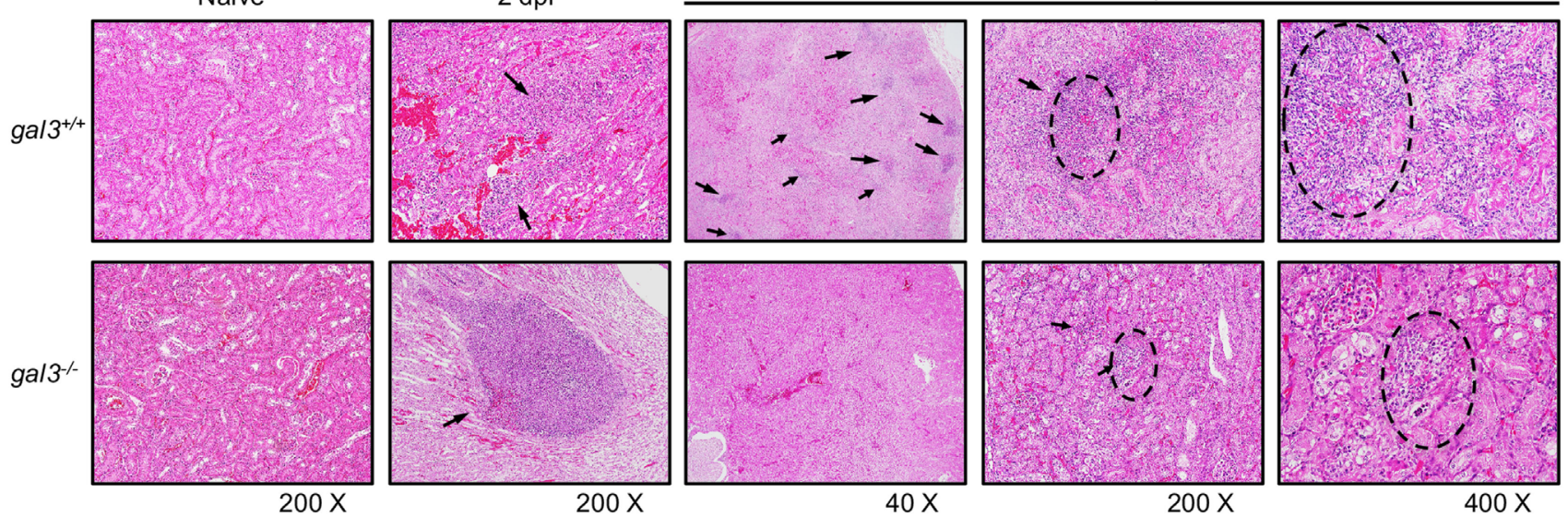

FIGURE 7 | Gal3 deficiency ameliorates Candida infection. Ga/3 ${ }^{+/+}$and ga/3-/- mice were infected intravenously with $5 \times 10^{5}$ of Candida. Kidneys were collected from uninfected (naïve) and infected mice at different time points after infection (dpi). (A) The survival of infected mice was followed until day 10 after infection. $n=11$. Data are pooled from three independent experiments. ${ }^{*} p<0.05$, as analyzed by log-rank and Fisher's exact test. (B) Fungal burden in the kidney is shown as $\log _{10}$ CFU per gram of tissue. $n=6$. Data are pooled from two independent experiments and presented as mean \pm SD. (C) Kidneys were collected from uninfected and infected mice on different days after infection (dpi). Left, arrows point to abscess lesions; right, kidney weight. $n=6$. Data are pooled from two independent experiments and presented as mean \pm SD. (D) The levels of blood urea nitrogen and creatinine in mouse sera on day 2 after infection. $n=5$. Data are pooled from two independent experiments. ${ }^{\star} p<0.05$; ${ }^{\star \star *} p<0.005$, as analyzed by Mann-Whitney test. (E) Kidneys were collected and fixed in formalin before H\&E stain. The magnification is $200 \times$ for sections from naïve mice and mice at day 2 after infection and 40x, 200x, and 400x for sections from mice at day 3 after infection. Arrows point to inflammatory foci. $n=3$. See also Figures S2 and S3 in Supplementary Material.

\section{The Negative Effect of gal3 on Neutrophil ROS Production Is Generalizable to Clinical C. albicans Isolates}

We tested C. albicans isolates from six patients with bloodstream candidiasis and showed that all six clinical isolates induced WT neutrophil ROS production (Figure 10A). Although the levels of ROS induced by different isolates varied, gal3 deficiency enhanced its production (Figure 10A). Isolates CL 15-1, CL 03-1, and CA 03-1, like SC5314, were more susceptible to killing by gal3 $^{-/-}$neutrophils than by gal3 $^{+/+}$neutrophils (Figure 10B), while isolates CL 10-1, CA 09-1, and CA 01-1 were highly and equally susceptible (>90\%) to killing by both $\mathrm{gal3}^{-/-}$and $\mathrm{gal3}^{+/+}$ neutrophils (Figure 10B). Consistent with the observation about 

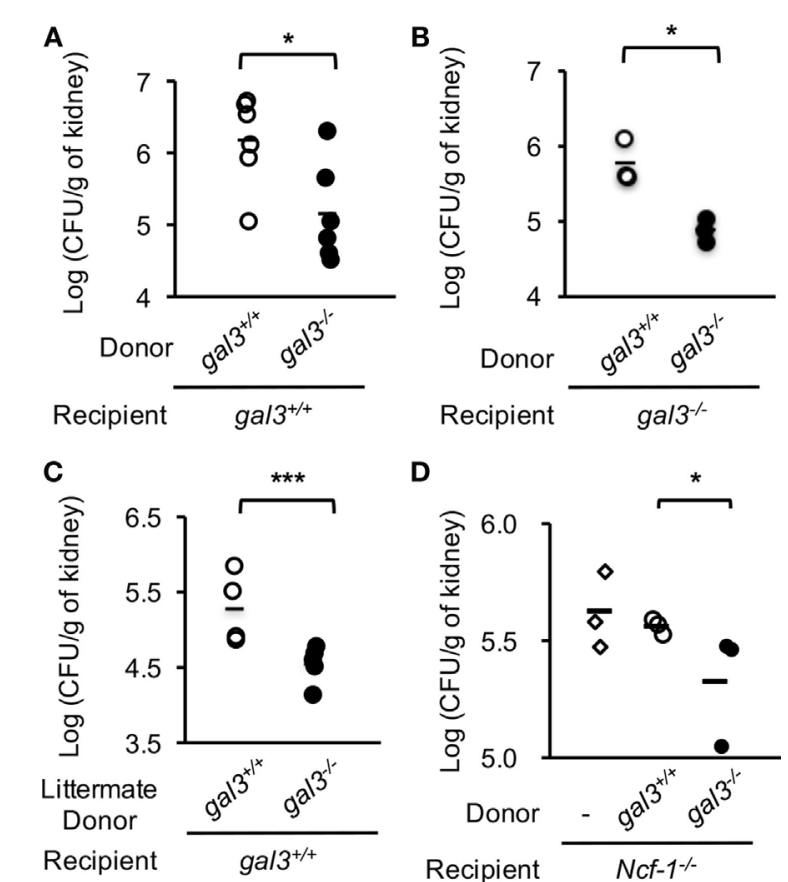

FIGURE 8 | Adoptive transfer of gal3 ${ }^{-/-}$neutrophils reduces fungal burden in recipient mice. Bone marrow neutrophils were collected from

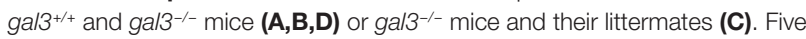

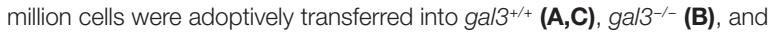
$n c f-1^{-/-}$(D) recipients separately. Recipient mice were infected with $5 \times 10^{5}$ (A-C) or $1 \times 10^{3}$ (D) of Candida yeast cells $1 \mathrm{~h}$ later. Fungal burdens in kidneys from recipient mice on 2 days after infection are shown as Log $_{10}$ (CFU per gram of kidney). $n=6$ pooled from two independent experiments in panel (A); $n=3$ in panel (B); $n=5$ in panel (C); and $n=3$ in panel (D) ${ }^{*} p<0.05 ;{ }^{* \star *} p<0.005$, as analyzed by Mann-Whitney test.

the role of gal3 in systemic SC5314 infection, gal3 $^{-/-}$mice were more efficient than $\mathrm{gal3}^{+/+}$mice in clearing CA 03-1 infection (Figure 10C) and had better survival (Figure 10D). Thus, gal3 negatively regulates neutrophil ROS production in response to not only SC5314 but also other clinical isolates and the negative effect of gal3 on neutrophil killing is more pronounced on otherwise "hard-to-kill" isolates.

\section{DISCUSSION}

In this study, we show that gal3 serves as a negative regulator of human and mouse neutrophil anti-Candida functions. Gal3 is expressed in the cytosol of both unstimulated human and mouse neutrophils. Responding to opsonized Candida stimulation, gal3 became detectable on the cell surface. While engulfed Candida is wrapped in phagosome (36), cytosolic gal3 does not directly contact the organism. Rather, it interacts with Syk and modulates its phosphorylation, through which it suppresses neutrophil anti-Candida function. Results of adoptive transfer experiments clearly demonstrates that cell intrinsic gal3 in neutrophils dampens host resistance to candidiasis. The negative effect of gal3 in primary human neutrophils is rescued by TD139 treatment and siRNA knockdown and demonstrable in neutrophil ROS response to clinical isolates of $C$. albicans. This study uncovers the immunomodulatory role of cell intrinsic gal3 in both human and mouse neutrophils and in host defense against systemic candidiasis in a mouse model.

Gal3 is proposed to be a biomarker for infectious disease (37). While the serum level of gal3 is low in healthy individuals, it is significantly elevated in patients with candidiasis compared to those with non-infectious inflammation (37). It appears that gal3 remains largely intracellular in healthy homeostatic condition and is released into circulation in systemic Candida infection. Since recombinant gal3 enhances human neutrophil phagocytosis of Candida parapsilosis yeasts and C. albicans hyphae (14), released gal3 in the body may enhance uptake of Candida by neutrophils. Here, we addressed cytosolic gal3 in anti-Candida functions and found that both human and mouse neutrophils constitutively express cytosolic gal3 and it is redistributed and becomes detectable on the surface after stimulation. Thus it seems unlikely that gal3 participates in neutrophil recognition of Candida. Employing $\beta$-lactose (up to $50 \mathrm{mM}$ ), a cell-impermeable pan-galectin inhibitor, we observed that neutrophil ROS production is not affected by the inhibitor after stimulation by opsonized Candida (unpublished data). Treatment with TD139, a cell-permeable gal3 inhibitor (F.-T. L., unpublished observation), however, increases neutrophil ROS production. Thus, it is cytosolic gal3 that negatively regulates neutrophil ROS production. Since neutrophils are of primary importance in defense against systemic candidiasis, the negative role of cytosolic gal3 in neutrophil anti-Candida functions cannot be ignored.

Cytosolic gal3 has been shown to bind to Alix, a subunit of the endosomal sorting complex, thereby promoting intracellular trafficking and surface expression of epidermal growth factor (EGF)-receptor in keratinocytes and enhancing EGF-induced migration (7). Gal3 is also known to serve as a binding partner of activated K-Ras, through which it enhances PI3K and Raf-1 activation (10). Gal3 binding to Bax protein in human thyroid carcinoma cells responding to apoptosis stimulus interferes with apoptosis (12). We show that cytosolic gal3 directly associates with Syk upon CR3 engagement. We reason that gal3 inhibits Syk phosphorylation through this interaction, thereby regulating a CR3 downstream signaling pathway. Syk being downstream of integrin engagement can be negatively regulated by $\mathrm{E} 3$ ligase $\mathrm{Cbl}$ resulting in degradation or by $\mathrm{SH} 2$ domain tyrosine phosphatase SHP-1 (38). Our data show that the levels of Syk protein in $\mathrm{gal}^{+/+}$ and $\mathrm{gal}^{-/-}$neutrophils remain unchanged after stimulation, suggesting that $\mathrm{Cbl}$ is not involved in gal3-mediated inhibition of Syk activation. It still awaits to be clarified whether gal 3 regulates Syk through recruitment of SHP-1 or through other mechanisms.

Gal3 has been shown to modulate immune response to infections in different animal models. In pulmonary Streptococcus pneumonia infection, the number of infiltrating neutrophils in the lungs of $\mathrm{gal3}^{-/-}$is lower than in $\mathrm{gal3}^{+/+}$mice (33). In experimental multiple sclerosis mouse model, gal3 ${ }^{-/}$mice infected with Theiler's murine encephalomyelitis virus have reduced CCL2, CCL5, CCL8, and CXCL10 expression and lower number of infiltrating cells in the brain subventricular zone (32). Infected with Trypanosoma cruzi, gal3 ${ }^{-/-}$mice exhibit higher 
A

Cytosolic gal3 Surface gal3

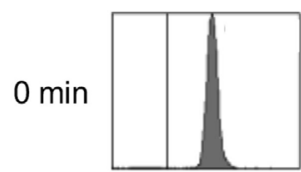

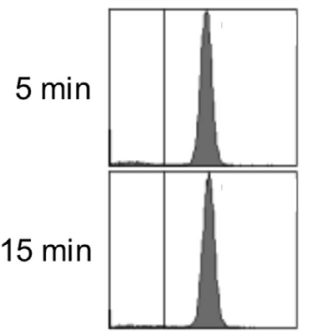

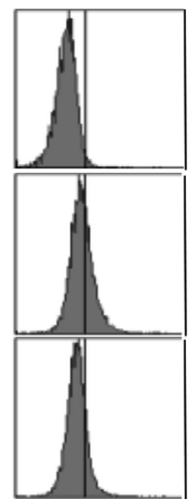

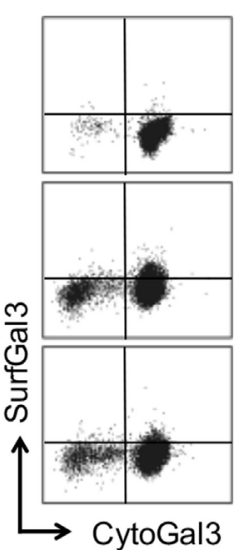

C

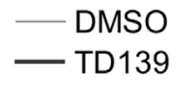

Gal3
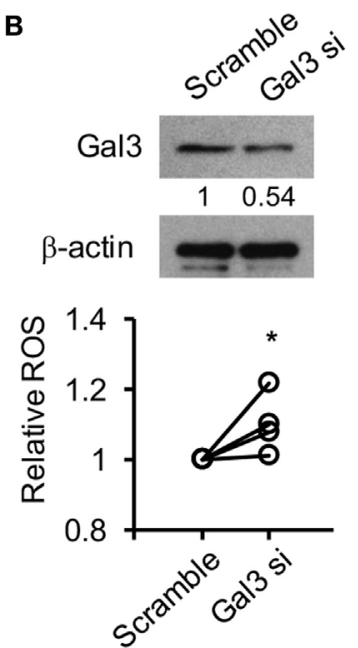

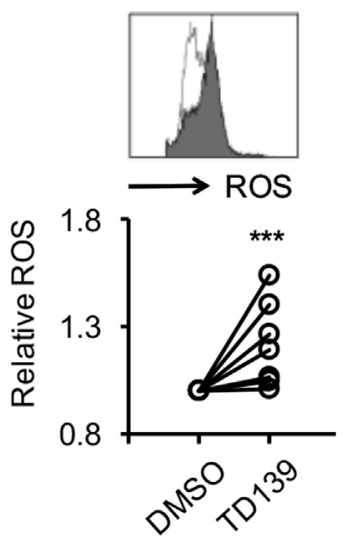

\begin{tabular}{|ccc|}
\hline & \multicolumn{2}{c}{ Cytosolic gal3 } \\
\cline { 2 - 3 } Ops.Ca & $\%$ & $\mathrm{MFI}$ \\
$0 \mathrm{~min}$ & 99.4 & 3824 \\
$5 \mathrm{~min}$ & 94.7 & 3084 \\
$15 \mathrm{~min}$ & 96.2 & 3541 \\
\hline & \multicolumn{2}{c}{ Surface gal3 } \\
\cline { 2 - 3 } Ops.Ca & $\%$ & $\mathrm{MFI}$ \\
$0 \mathrm{~min}$ & 1.7 & 152 \\
$5 \mathrm{~min}$ & 36.4 & 280 \\
$15 \mathrm{~min}$ & 17.7 & 229 \\
\hline
\end{tabular}

D

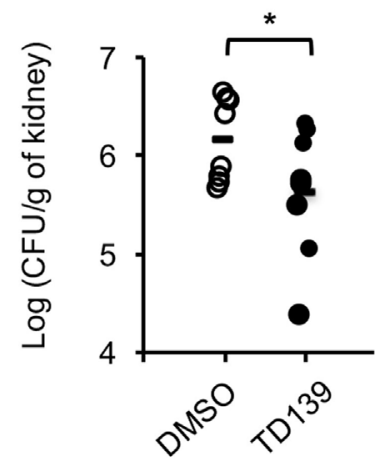

FIGURE 9 | Reducing gal3 expression or activity enhances human neutrophil reactive oxygen species (ROS) response to opsonized Candida and host resistance to Candida infection. (A-C) Human neutrophils isolated from peripheral blood of healthy volunteers were allowed to ingest opsonized (in 5\% human serum) Candida yeasts. (A) The \% of cells expressing cytosolic gal3 ${ }^{+}$or surface gal3 ${ }^{+}$in total cells. (B,C) Cells were transfected with gal3 (Gal3 si) or scrambled (Scramble) siRNA (B) or pretreated with mock (0.5\% DMSO) or $250 \mu \mathrm{M}$ of TD139 for $4 \mathrm{~h}$ (C) before stimulation with opsonized Candida. The level of ROS production by neutrophils was analyzed by flow cytometry at 20 min after stimulation. Relative ROS production was calculated by dividing the mean fluorescence intensity (MFI) of cells treated with Gal3 siRNA TD139 and by that treated with scrambled siRNA and in DMSO, respectively. The levels of gal3 protein in cells transfected with gal3 or scrambled siRNA were detected by Western blot to determine the efficiency of gal3 siRNA knockdown. $\beta$-actin was used as a loading control. The numbers below the gel denote the relative intensity of gal3. (D) Ga/3 ${ }^{+/+}$mice were infected with $5 \times 10^{5}$ of Candida yeast cells intravenously. After 30 min, infected mice were injected with 1.5\% DMSO (DMSO) or $300 \mu \mathrm{g}$ of TD139 in $200 \mu \mathrm{l}$ of RPMl medium intraperitoneally. Fungal burdens in kidneys from infected mice on 2 days after infection are shown as $\log _{10}$ (CFU per gram of kidney) $n=4$ in panel (B); $n=7$ in panel (C); and $n=8$ in panel (D). ${ }^{*} p<0.05$; ${ }^{\star \star \star} p<0.005$, as analyzed by Mann-Whitney test.

levels of parasitemia but have reduced cellular infiltration and tissue damage in the heart (39). Systemic infection by C. albicans results in inflammation-induced renal pathology leading to death while $\mathrm{Crr}^{-/-}$or Ifnarl ${ }^{-/-}$mice have reduced number of renal infiltrating cells and develop less severe renal pathology and better survival $(40,41)$, suggesting that neutrophil infiltration in systemic candidiasis denotes poor prognosis. Although studies of bacterial and parasitic infections demonstrate that the presence of gal3 affects neutrophil infiltration, we observed in this study that while the expression of neutrophil-attracting chemokines and renal neutrophil infiltration are comparable in $\mathrm{gal3}^{+/+}$and gal3 $^{-/-}$mice after systemic Candida infection, gal3 $3^{-/-}$mice have less renal pathology and better survival than gal3 $^{+/+}$mice. Renal infiltrating neutrophils from gal3 $^{-/-}$mice produce higher level of ROS and kill Candida more efficiently. Thus, gal3 functions to inhibit neutrophil ROS production and the ability of neutrophils to kill Candida in systemic candidiasis rather than positively modulate cellular infiltration like that in bacterial and parasitic infections.

Innate cells other than neutrophils are also known to be involved in protecting the host from Candida infection. Depleting monocyte/macrophage by clodronate-containing 


\section{A}
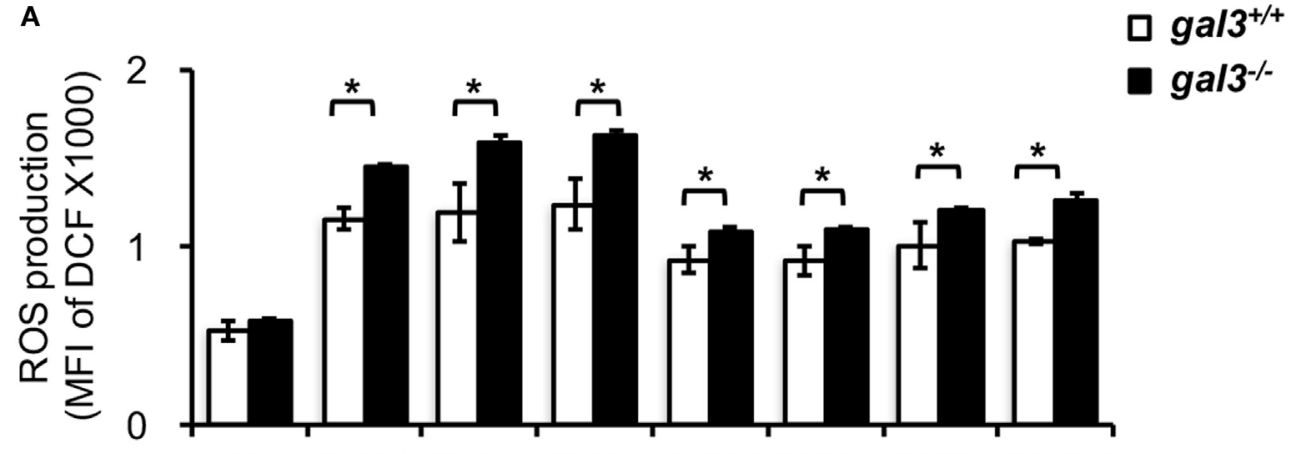

Un CL10-1 CA09-1 CL15-1 CA01-1 CL03-1 CA03-1 SC5314

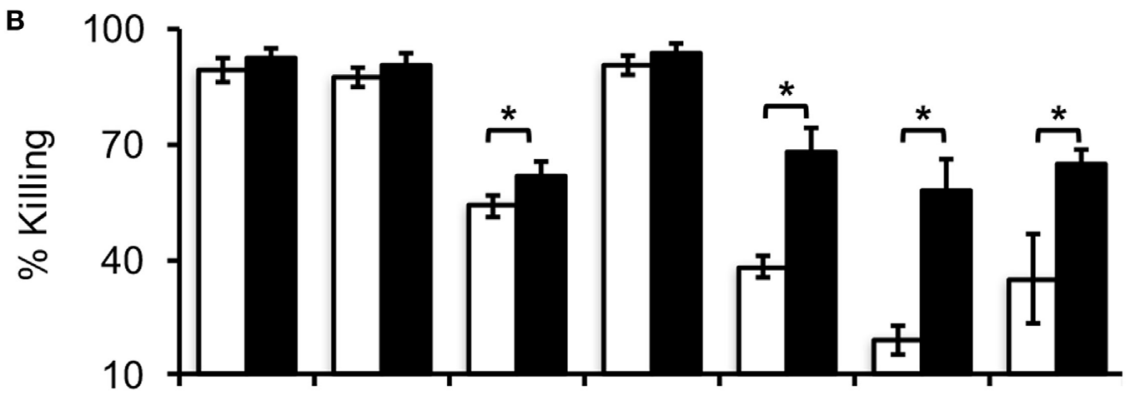

CL10-1 CA09-1 CL15-1 CA01-1 CL03-1 CA03-1 SC5314

C

$$
2 \text { d.p.i. }
$$

$\left(1 \times 10^{6} \mathrm{CA} 03-1\right)$

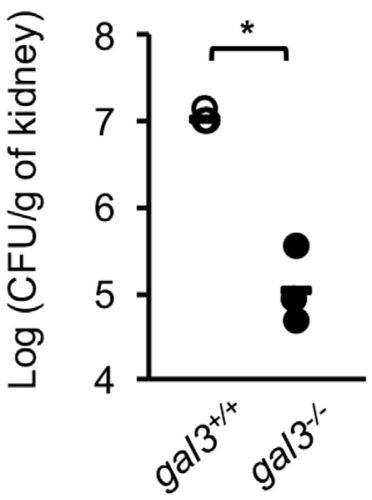

D
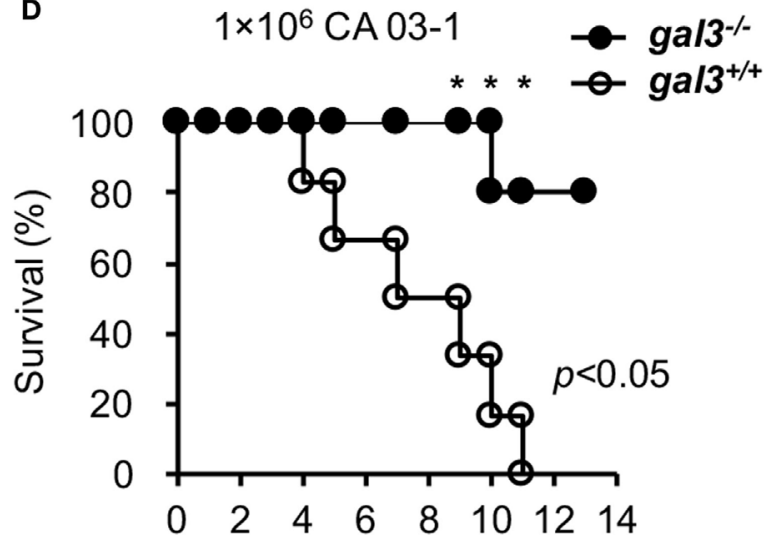

Time after infection (day)

FIGURE 10 | The effect of gal3 on neutrophil response to Candida albicans clinical isolates. (A,B) Bone marrow neutrophils isolated from ga/3 ${ }^{+/+}$and ga/3-/- mice were allowed to ingest opsonized C. albicans clinical isolates CL 10-1, CA 09-1, CL 15-1, CA 01-1, CL 03-1, CA 03-1, and SC 5314. (A) The levels of reactive oxygen species (ROS) production are shown as mean fluorescence intensity (MFI) of oxidized DCF fluorescence. $n=4$. (B) The abilities of neutrophils to kill opsonized Candida are shown as \% killing. $n=4$. Data are presented as mean \pm SD. ${ }^{*} p<0.05$, as analyzed by Mann-Whitney test. (C,D) Ga/3+/+ and ga/3-/- mice were infected intravenously with $1 \times 10^{6}$ of clinical isolate CA 03-1. (C) Fungal burdens in kidneys on day 2 after infection are presented as Log 10 (CFU per gram of kidney). Ga/3 ${ }^{+/+}, n=4$; and ga/3 ${ }^{-/-}, n=3$. * $p<0.05$, as analyzed by Mann-Whitney test. (D) The survival of infected mice was followed until day 10 after infection. Gal ${ }^{+/+}, n=6$; and ga/3 ${ }^{-/-}, n=5 .{ }^{*} p<0.05$, as analyzed by log-rank and Fisher's exact test.

liposomes abolishes host resistance to systemic candidiasis (42). Depleting CCR2 ${ }^{+}$cells interrupts monocyte infiltration and increases fungal burdens in kidney, brain, and spleen during early phase of Candida infection (43). Reduced number of renal infiltrating macrophages in infected $\mathrm{CX}_{3} \mathrm{CR} 1$-deficient mice significantly exacerbates systemic candidiasis (34). Moreover, E3 ubiquitin ligase CBLB protein negatively regulates macrophage and dendritic cell anti-Candida functions with $\mathrm{Cblb}^{-/-}$mice more resistant to Candida infection (44). Knocking out Syk in dendritic cells with CD11c promoter-driven Syk-deletion demonstrates that dendritic cells contribute to clearance of Candida through production of IL-23 which is important to NK cell secretion of GM-CSF (45). NK cell-derived GM-CSF promotes neutrophil ROS production and fungicidal activity (46). 
We discovered that in addition to regulation of neutrophil ROS production, endogenous gal 3 was also involved in macrophage activation, monocyte, and dendritic cell recruitment to kidneys in systemic candidiasis. Consistent with previous findings (47, 48 ), we found that gal3 plays a positive role in activation of M2 macrophage marked by lower MHC II and higher CD206 expression. Moreover, the negative effect of gal3 is abolished by depleting either neutrophils or macrophages (Figures S6A,B in Supplementary Material). These results show that gal3 is involved not only in neutrophil but also other myeloid cell anti-Candida immune response. While we delineated the mechanism of how gal3 regulates neutrophil ROS production through suppression of Syk activation, the mechanism of how gal3 regulates macrophages and dendritic cells in systemic candidiasis warrants further study.

While our study reveals the negative role of gal3 in systemic infection with Candida $\left(5 \times 10^{5} \mathrm{CFU}\right)$, Linden et al. reported an opposite effect (49). In their study, gal3 $^{+/+}$mice infected with low lethal dose of Candida $\left(1.0 \times 10^{5} \mathrm{CFU}\right)$ have fewer abscesses in the kidneys, lower fungal burdens and better survival than $\mathrm{gal3}^{-/-}$ mice. Exaggerated inflammatory infiltration and uncontrolled inflammatory responses were attributed to higher mortality in gal3 $^{-/-}$mice. Previous study employing Ifnar $1^{-/-}$mice showed opposite effects of type I interferon in host responses to high lethal $\left(5.0 \times 10^{5} \mathrm{CFU}\right)$, low lethal $\left(1.0 \times 10^{5} \mathrm{CFU}\right)$, and sublethal $\left(5.0 \times 10^{4} \mathrm{CFU}\right)$ doses of Candida challenge (41). We speculate that different sizes of inoculum, different commensal flora in the mice resulting from different housing conditions, as well as the difference in the embryonic stem cells (WW6 ES cells vs. D3 ES cells) employed and the backcross strategy may account for our opposite observations. However, our adoptive transfer experiments showing that transferred gal3 $3^{-/-}$neutrophils clear Candida more efficiently in recipient mice than $\mathrm{gal}^{+/+}$neutrophils support our conclusion that cell intrinsic gal 3 negatively regulates host defense against systemic candidiasis.

Our results show that gal3 regulates both human and mouse neutrophil ROS production and that the efficiency of neutrophils to kill Candida varies depending on the isolates they encountered. Gal3 deficiency enhances the ability of mouse neutrophils to kill when encountering "hard-to-kill" Candida. Based on galectin CRD binding to $\beta$-galactoside, Nilsson and colleagues developed a novel gal3 antagonist, TD139 (50). While TD139 binds to gal1, 3 and 7 , its binding affinity to gal 3 is much higher than binding to gal7 and gal1 (27). Since TD139 can get inside the cell, our results showing this compound inhibits neutrophil ROS production suggest that TD139 functions intracellularly to interfere gal3 and Syk association. Treatment with TD139 has been reported to reduce disease severity in animal models of idiopathic pulmonary fibrosis (51), Concanavalin A-induced hepatitis (52), and type 1 diabetes (53). These studies demonstrated a potential treatment strategy for using TD139 in diseases where gal3 contributes positively to pathogenesis. Phase I clinical trial has demonstrated that TD139 is safe and tolerable when used in healthy volunteers (http://ClinicalTrials.gov identifier, NCT02257177). Thus, using TD139 to block the activity of gal3 is a viable option for enhancing neutrophil killing of Candida possibly when used in combination with antifungal drug treatment.
In summary, this study shows that the dynamics of gal3 in cytosol changes after neutrophils encountering Candida. Gal3 which physically interacts with Syk negatively affects CR3 downstream Syk phosphorylation and thus negatively regulates Syk-mediated ROS production. We also demonstrate in adoptive transfer experiments that cell intrinsic gal3 in neutrophils dampens host defense against candidiasis. The effect of gal3 in neutrophil interaction with $C$. albicans is generalizable to "hard-to-kill" clinical isolates. This study uncovers the new role of cytosolic gal3 in binding to Syk and in modulating CR3 downstream signal pathway in response to Candida. Importantly, treatment with antagonist TD139 and silencing gal3 expression in human neutrophils enhances neutrophil anti-Candida function. Our study points to the possibility of targeting gal3 as a potential therapeutic strategy for controlling systemic candidiasis.

\section{ACCESSION NUMBERS}

The accession numbers in the UniPortKB/SwissProt database of the proteins mentioned in this study are follows: mouse gal3, P16110; human gal3, P17931; CD11b, P05555; Syk, P43404; and NCF-1, Q09014.

\section{AUTHOR CONTRIBUTIONS}

S-YW contributed to the conception and design of the work; devoted to the acquisition, analysis, and interpretation of data for the work; drafted the work; and revised the manuscript critically for important intellectual content. J-HH and W-YC devoted to the acquisition, analysis, and interpretation of data for the work and drafted the work. Y-C Chen, Y-C Chan, and $\mathrm{C}-\mathrm{HL}$ contributed to the conception and design of the work and provided reagents that are required resolving questions related to the integrity of the work. F-TL contributed to the conception and design of the work, provided reagents, and revised the manuscript critically for important intellectual content. BW-H contributed to the conception and design of the work, devoted to the interpretation of data for the work, drafted the work, revised the manuscript critically for important intellectual content, final approval of the version to be published, and agreed to be accountable for all aspects of the work in ensuring that questions related to the accuracy or integrity of any part of the work are appropriately investigated and resolved.

\section{ACKNOWLEDGMENTS}

The authors thank Dr. Gordon Brown (University of Aberdeen, $\mathrm{UK})$ for gift of $\mathrm{Clec} \mathrm{a}^{-/-}$mice. The authors are grateful to Dr. Chung-Yu Lan (National Tsing Hua University, Taiwan) for the gift of GFP-expressing Candida strain OG1. The services provided by the Flow Cytometric Analyzing and Sorting Core and the Imaging Core at the First Core Lab of National Taiwan University College of Medicine and the Second Core Lab of Department of Medical Research, National Taiwan University Hospital are gratefully acknowledged. The authors also thank the National Laboratory Animal Center (NLAC) of the National 
Applied Research Laboratories, Taiwan, for histopathological evaluation of $\mathrm{H} \& \mathrm{E}$ sections.

\section{FUNDING}

The work was supported by Academia Sinica (https://www. sinica.edu.tw/index.shtml) Thematic Research Program AS-105TP-B08-3 and grant 104-2320-B-002-052-MY2 (to BW-H) from the Ministry of Science and Technology (MOST, https://www. most.gov.tw/ch/academic), grants 102-2314-B-002-158-MY3

\section{REFERENCES}

1. Dumic J, Dabelic S, Flogel M. Galectin-3: an open-ended story. Biochim Biophys Acta (2006) 1760(4):616-35. doi:10.1016/j.bbagen.2005.12.020

2. Rabinovich GA, Toscano MA. Turning 'sweet' on immunity: galectin-glycan interactions in immune tolerance and inflammation. Nat Rev Immunol (2009) 9(5):338-52. doi:10.1038/nri2536

3. Sundblad V, Croci DO, Rabinovich GA. Regulated expression of galectin-3, a multifunctional glycan-binding protein, in haematopoietic and non-haematopoietic tissues. Histol Histopathol (2011) 26(2):247-65. doi:10.14670/ HH-26.247

4. Alves CM, Silva DA, Azzolini AE, Marzocchi-Machado CM, Carvalho JV, Pajuaba AC, et al. Galectin-3 plays a modulatory role in the life span and activation of murine neutrophils during early Toxoplasma gondii infection. Immunobiology (2010) 215(6):475-85. doi:10.1016/j.imbio.2009.08.001

5. Chen HY, Fermin A, Vardhana S, Weng IC, Lo KF, Chang EY, et al. Galectin-3 negatively regulates TCR-mediated CD4+ T-cell activation at the immunological synapse. Proc Natl Acad Sci U S A (2009) 106(34):14496-501. doi:10.1073/ pnas.0903497106

6. Ferraz LC, Bernardes ES, Oliveira AF, Ruas LP, Fermino ML, Soares SG, et al. Lack of galectin-3 alters the balance of innate immune cytokines and confers resistance to Rhodococcus equi infection. Eur JImmunol (2008) 38(10):2762-75. doi:10.1002/eji.200737986

7. Liu W, Hsu DK, Chen HY, Yang RY, Carraway KL III, Isseroff RR, et al. Galectin-3 regulates intracellular trafficking of EGFR through Alix and promotes keratinocyte migration. J Invest Dermatol (2012) 132(12):2828-37. doi:10.1038/jid.2012.211

8. Nakahara S, Oka N, Raz A. On the role of galectin-3 in cancer apoptosis. Apoptosis (2005) 10(2):267-75. doi:10.1007/s10495-005-0801-y

9. Esteban A, Popp MW, Vyas VK, Strijbis K, Ploegh HL, Fink GR. Fungal recognition is mediated by the association of dectin- 1 and galectin- 3 in macrophages. Proc Natl Acad Sci U S A (2011) 108(34):14270-5. doi:10.1073/ pnas. 1111415108

10. Elad-Sfadia G, Haklai R, Balan E, Kloog Y. Galectin-3 augments K-Ras activation and triggers a Ras signal that attenuates ERK but not phosphoinositide 3-kinase activity. J Biol Chem (2004) 279(33):34922-30. doi:10.1074/jbc. M312697200

11. Li Y, Liu L, Niu Y, Feng J, Sun Y, Kong X, et al. Modified apple polysaccharide prevents against tumorigenesis in a mouse model of colitis-associated colon cancer: role of galectin-3 and apoptosis in cancer prevention. Eur J Nutr (2012) 51(1):107-17. doi:10.1007/s00394-011-0194-3

12. Harazono Y, Kho DH, Balan V, Nakajima K, Zhang T, Hogan V, et al. Galectin-3 leads to attenuation of apoptosis through Bax heterodimerization in human thyroid carcinoma cells. Oncotarget (2014) 5(20):9992-10001. doi:10.18632/ oncotarget. 2486

13. Farnworth SL, Henderson NC, Mackinnon AC, Atkinson KM, Wilkinson T, Dhaliwal K, et al. Galectin-3 reduces the severity of pneumococcal pneumonia by augmenting neutrophil function. Am J Pathol (2008) 172(2):395-405. doi:10.2353/ajpath.2008.070870

14. Linden JR, Kunkel D, Laforce-Nesbitt SS, Bliss JM. The role of galectin-3 in phagocytosis of Candida albicans and Candida parapsilosis by human neutrophils. Cell Microbiol (2013) 15(7):1127-42. doi:10.1111/cmi.12103

15. Nieminen J, St-Pierre C, Sato S. Galectin-3 interacts with naive and primed neutrophils, inducing innate immune responses. JLeukoc Biol (2005) 78(5):1127-35. doi:10.1189/jlb.1204702 and 104-2314-B-002-241 (to Y.-Y. Chen) from MOST, funding from the Summit Program at Academia Sinica (to FTL and CHL), AS-105-TP-B08-1 (to FTL) and grants 104-0210-01-09-02, 105-0210-01-13-02, 106-0210-01-15-02 from MOST (to CHL).

\section{SUPPLEMENTARY MATERIAL}

The Supplementary Material for this article can be found online at http://journal.frontiersin.org/article/10.3389/fimmu. 2017.00048/full\#supplementary-material.

16. Gow NA, van de Veerdonk FL, Brown AJ, Netea MG. Candida albicans morphogenesis and host defence: discriminating invasion from colonization. Nat Rev Microbiol (2012) 10(2):112-22. doi:10.1038/nrmicro2711

17. Antachopoulos C, Walsh TJ, Roilides E. Fungal infections in primary immunodeficiencies. Eur J Pediatr (2007) 166(11):1099-117. doi:10.1007/ s00431-007-0527-7

18. Horn DL, Neofytos D, Anaissie EJ, Fishman JA, Steinbach WJ, Olyaei AJ, et al. Epidemiology and outcomes of candidemia in 2019 patients: data from the prospective antifungal therapy alliance registry. Clin Infect Dis (2009) 48(12):1695-703. doi:10.1086/599039

19. Lionakis MS, Netea MG. Candida and host determinants of susceptibility to invasive candidiasis. PLoS Pathog (2013) 9(1):e1003079. doi:10.1371/journal. ppat.1003079

20. Kullberg BJ, Arendrup MC. Invasive candidiasis. N Engl J Med (2015) 373(15):1445-56. doi:10.1056/NEJMra1315399

21. Chen PY, Chuang YC, Wang JT, Sheng WH, Yu CJ, Chu CC, et al. Comparison of epidemiology and treatment outcome of patients with candidemia at a teaching hospital in Northern Taiwan, in 2002 and 2010. J Microbiol Immunol Infect (2014) 47(2):95-103. doi:10.1016/j.jmii.2012.08.025

22. Lionakis MS, Lim JK, Lee CC, Murphy PM. Organ-specific innate immune responses in a mouse model of invasive candidiasis. J Innate Immun (2011) 3(2):180-99. doi:10.1159/000321157

23. Aratani Y, Kura F, Watanabe H, Akagawa H, Takano Y, Suzuki K, et al. Critical role of myeloperoxidase and nicotinamide adenine dinucleotide phosphate-oxidase in high-burden systemic infection of mice with Candida albicans. J Infect Dis (2002) 185(12):1833-7. doi:10.1086/340635

24. Koh AY, Kohler JR, Coggshall KT, Van Rooijen N, Pier GB. Mucosal damage and neutropenia are required for Candida albicans dissemination. PLoS Pathog (2008) 4(2):e35. doi:10.1371/journal.ppat.0040035

25. Chao CC, Hsu PC, Jen CF, Chen IH, Wang CH, Chan HC, et al. Zebrafish as a model host for Candida albicans infection. Infect Immun (2010) 78(6):2512-21. doi:10.1128/IAI.01293-09

26. Maqbool M, Vidyadaran S, George E, Ramasamy R. Optimisation of laboratory procedures for isolating human peripheral blood derived neutrophils. Med J Malaysia (2011) 66(4):296-9.

27. Hsieh TJ, Lin HY, Tu Z, Lin TC, Wu SC, Tseng YY, et al. Dual thio-digalactoside-binding modes of human galectins as the structural basis for the design of potent and selective inhibitors. Sci Rep (2016) 6:29457. doi:10.1038/srep29457

28. Vonk AG, Netea MG, Kullberg BJ. Phagocytosis and intracellular killing of Candida albicans by murine polymorphonuclear neutrophils. Methods Mol Biol (2012) 845:277-87. doi:10.1007/978-1-61779-539-8_18

29. Novak R, Dabelic S, Dumic J. Galectin-1 and galectin-3 expression profiles in classically and alternatively activated human macrophages. Biochim Biophys Acta (2012) 1820(9):1383-90. doi:10.1016/j.bbagen.2011.11.014

30. Shan M, Gentile M, Yeiser JR, Walland AC, Bornstein VU, Chen K, et al. Mucus enhances gut homeostasis and oral tolerance by delivering immunoregulatory signals. Science (2013) 342(6157):447-53. doi:10.1126/science.1237910

31. Gazendam RP, van Hamme JL, Tool AT, van Houdt M, Verkuijlen PJ, Herbst $\mathrm{M}$, et al. Two independent killing mechanisms of Candida albicans by human neutrophils: evidence from innate immunity defects. Blood (2014) 124(4):590-7. doi:10.1182/blood-2014-01-551473

32. James RE, Hillis J, Adorjan I, Gration B, Mundim MV, Iqbal AJ, et al. Loss of galectin-3 decreases the number of immune cells in the subventricular zone and restores proliferation in a viral model of multiple sclerosis. Glia (2016) 64(1):105-21. doi:10.1002/glia.22906 
33. Nieminen J, St-Pierre C, Bhaumik P, Poirier F, Sato S. Role of galectin-3 in leukocyte recruitment in a murine model of lung infection by Streptococcus pneumoniae. J Immunol (2008) 180(4):2466-73. doi:10.4049/jimmunol.180.4.2466

34. Lionakis MS, Swamydas M, Fischer BG, Plantinga TS, Johnson MD, Jaeger $\mathrm{M}$, et al. CX3CR1-dependent renal macrophage survival promotes Candida control and host survival. J Clin Invest (2013) 123(12):5035-51. doi:10.1172/ JCI71307

35. Chen Y, Mendoza S, Davis-Gorman G, Cohen Z, Gonzales R, Tuttle H, et al. Neutrophil activation by murine retroviral infection during chronic ethanol consumption. Alcohol Alcohol (2003) 38(2):109-14. doi:10.1093/alcalc/agg049

36. Bain JM, Louw J, Lewis LE, Okai B, Walls CA, Ballou ER, et al. Candida albicans hypha formation and mannan masking of beta-glucan inhibit macrophage phagosome maturation. MBio (2014) 5(6):e01874. doi:10.1128/ mBio.01874-14

37. ten Oever J, Giamarellos-Bourboulis EJ, van de Veerdonk FL, Stelma FF, Simon A, Janssen M, et al. Circulating galectin-3 in infections and non-infectious inflammatory diseases. Eur J Clin Microbiol Infect Dis (2013) 32(12):1605-10. doi:10.1007/s10096-013-1919-4

38. Mocsai A, Ruland J, Tybulewicz VL. The SYK tyrosine kinase: a crucial player in diverse biological functions. Nat Rev Immunol (2010) 10(6):387-402. doi: $10.1038 /$ nri2765

39. Pineda MA, Cuervo H, Fresno M, Soto M, Bonay P. Lack of galectin-3 prevents cardiac fibrosis and effective immune responses in a murine model of Trypanosoma cruzi infection. J Infect Dis (2015) 212(7):1160-71. doi:10.1093/ infdis/jiv185

40. Lionakis MS, Fischer BG, Lim JK, Swamydas M, Wan W, Richard Lee CC, et al. Chemokine receptor Ccrl drives neutrophil-mediated kidney immunopathology and mortality in invasive candidiasis. PLoS Pathog (2012) 8(8):e1002865. doi:10.1371/journal.ppat.1002865

41. Majer O, Bourgeois C, Zwolanek F, Lassnig C, Kerjaschki D, Mack M, et al. Type I interferons promote fatal immunopathology by regulating inflammatory monocytes and neutrophils during Candida infections. PLoS Pathog (2012) 8(7):e1002811. doi:10.1371/journal.ppat.1002811

42. Qian Q, Jutila MA, Van Rooijen N, Cutler JE. Elimination of mouse splenic macrophages correlates with increased susceptibility to experimental disseminated candidiasis. J Immunol (1994) 152(10):5000-8.

43. Ngo LY, Kasahara S, Kumasaka DK, Knoblaugh SE, Jhingran A, Hohl TM. Inflammatory monocytes mediate early and organ-specific innate defense during systemic candidiasis. J Infect Dis (2014) 209(1):109-19. doi:10.1093/ infdis/jit413

44. Wirnsberger G, Zwolanek F, Asaoka T, Kozieradzki I, Tortola L, Wimmer RA, et al. Inhibition of CBLB protects from lethal Candida albicans sepsis. Nat Med (2016) 22(8):915-23. doi:10.1038/nm.4134
45. Whitney PG, Bar E, Osorio F, Rogers NC, Schraml BU, Deddouche S, et al Syk signaling in dendritic cells orchestrates innate resistance to systemic fungal infection. PLoS Pathog (2014) 10(7):e1004276. doi:10.1371/journal. ppat.1004276

46. Bar E, Whitney PG, Moor K, Reis e Sousa C, LeibundGut-Landmann S. IL-17 regulates systemic fungal immunity by controlling the functional competence of NK cells. Immunity (2014) 40(1):117-27. doi:10.1016/j. immuni.2013.12.002

47. MacKinnon AC, Farnworth SL, Hodkinson PS, Henderson NC, Atkinson KM, Leffler $\mathrm{H}$, et al. Regulation of alternative macrophage activation by galectin-3. J Immunol (2008) 180(4):2650-8. doi:10.4049/ jimmunol.180.4.2650

48. Corna G, Campana L, Pignatti E, Castiglioni A, Tagliafico E, Bosurgi L, et al. Polarization dictates iron handling by inflammatory and alternatively activated macrophages. Haematologica (2010) 95(11):1814-22. doi:10.3324/ haematol.2010.023879

49. Linden JR, De Paepe ME, Laforce-Nesbitt SS, Bliss JM. Galectin-3 plays an important role in protection against disseminated candidiasis. Med Mycol (2013) 51(6):641-51. doi:10.3109/13693786.2013.770607

50. Nilsson U, Leffler H, Mukhopadhyay B, Rajput V, Inventor; Galecto Biotech Ab, Assignee. Galectoside Inhibitors of Galectins. US patent 9353141 (2016)

51. Mackinnon AC, Gibbons MA, Farnworth SL, Leffler H, Nilsson UJ, Delaine $\mathrm{T}$, et al. Regulation of transforming growth factor-betal-driven lung fibrosis by galectin-3. Am J Respir Crit Care Med (2012) 185(5):537-46. doi:10.1164/ rccm.201106-0965OC

52. Volarevic V, Milovanovic M, Ljujic B, Pejnovic N, Arsenijevic N, Nilsson U, et al. Galectin-3 deficiency prevents concanavalin A-induced hepatitis in mice. Hepatology (2012) 55(6):1954-64. doi:10.1002/hep.25542

53. Saksida T, Nikolic I, Vujicic M, Nilsson UJ, Leffler H, Lukic ML, et al. Galectin-3 deficiency protects pancreatic islet cells from cytokine-triggered apoptosis in vitro. J Cell Physiol (2013) 228(7):1568-76. doi:10.1002/jcp.24318

Conflict of Interest Statement: The authors declare that the research was conducted in the absence of any commercial or financial relationships that could be construed as a potential conflict of interest.

Copyright (C) 2017 Wu, Huang, Chen, Chan, Lin, Chen, Liu and Wu-Hsieh. This is an open-access article distributed under the terms of the Creative Commons Attribution License (CC BY). The use, distribution or reproduction in other forums is permitted, provided the original author(s) or licensor are credited and that the original publication in this journal is cited, in accordance with accepted academic practice. No use, distribution or reproduction is permitted which does not comply with these terms. 